\title{
Nonuniform and Higher-order FDTD Methods for the Schrödinger Equation
}

\author{
Pieter Decleer ${ }^{\mathrm{a}, *}$, Arne Van Londersele ${ }^{\mathrm{a}}$, Hendrik Rogier ${ }^{\mathrm{a}}$, Dries Vande Ginste ${ }^{\mathrm{a}}$ \\ ${ }^{a}$ Electromagnetics Group/IDLab, Department of Information Technology, Ghent University/imec, \\ Technologiepark-Zwijnaarde 126, Ghent, Belgium
}

\begin{abstract}
Two Finite-Difference Time-Domain (FDTD) methods are developed for solving the Schrödinger equation on nonuniform tensor-product grids. The first is an extension of the standard second-order accurate spatial differencing scheme on uniform grids to nonuniform grids, whereas the second utilizes a higher-order accurate spatial scheme using an extended stencil. Based on discrete-time stability theory, an upper bound is derived for the time step of both proposed schemes. It is shown that the time step derived in this way can be larger compared to the known stability criterion. Furthermore, the numerical dispersion error is investigated as a function of the time step, the spatial step and the propagation direction. Numerical experiments are compared with analytical solutions and demonstrate the increased accuracy of the higher-order scheme as well as the advantageous properties of nonuniform gridding.
\end{abstract}

Keywords: Finite-difference time-domain (FDTD), Schrödinger equation, Stability, Nonuniform, Higher-order schemes

\section{Introduction}

Describing the time-dependent behavior of charged particles inside molecular structures is of great importance in the description of nanoscale devices. Therefore, it is essential to develop efficient and accurate tools capable of solving the time-dependent Schrödinger equation. The Finite-Difference Time-Domain (FDTD) method is a well-known technique that discretizes the wave function on a space-time structured grid [1-9]. Depending on the discretization of the temporal derivative, the resulting scheme is either implicit or explicit. The former can be unconditionally stable but has the drawback of requiring expensive matrix inversions, whereas the latter only requires cheap arithmetic operations but suffers from small time steps to ensure stability. The linear numerical complexity of explicit methods often makes them favorable over implicit methods when it comes to computationally large problems.

To lower the computational cost pertaining to explicit schemes, obviously, one can reduce the number of spatial variables or the number of time iterations. Higher-order accurate spatial schemes employ coarser grids that effectuate both at the same time, as a coarser grid is used, which in turn support a larger time step. These higher-order accurate spatial schemes are highly performant because they can be interpreted as a sum-accelerated pseudospectral method [10].

Complex molecular structures require large grids with very small spatial steps, resulting in an unreasonable amount of spatial variables. The high accuracy, however, is only required in specific areas of the structure, e.g. the atoms. To increase the accuracy in these areas, it is possible to use nonuniform tensor product grids, meaning that each spatial step can vary along its axis. In [11], such a scheme is proposed, where a mapping function is used to convert a nonuniform grid into a uniform grid. However, it does not allow for true higher-order accurate spatial schemes even when using extended stencils. The combination of a true higher-order spatial scheme with nonuniform grids is still lacking, as is a detailed discussion on the influence

\footnotetext{
* Corresponding author

Email address: pieter.decleer@ugent.be (Pieter Decleer)
} 


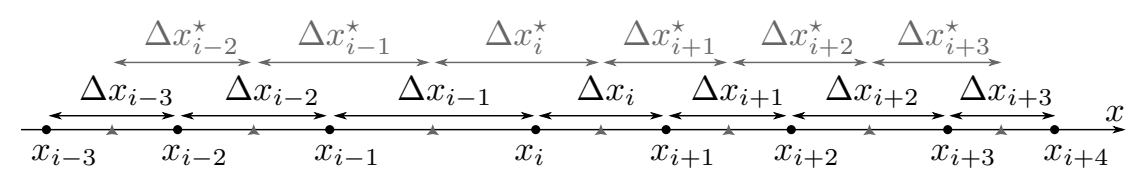

Figure 1: Definition of the nonuniform spatial grid variables in the $x$-direction. The positions of the primary grid points $x_{i}$ are denoted by dots and the positions of the dual grid points are denoted by the pointed triangles. The primary grid steps are defined as the distance between two primary grid points and the dual grid steps are defined as $\Delta x_{i}^{\star}=\left(\Delta x_{i}+\Delta x_{i-1}\right) / 2$.

on the stability of the resulting FDTD scheme.

In this paper, two explicit schemes are proposed that solve the Schrödinger equation on a nonuniform grid without explicitly needing a mapping function and, as such, allow for much more flexibility in the used grid. The first scheme modifies the second-order accurate central differencing scheme to a nonuniform grid by introducing a dual grid. The second scheme is higher-order accurate, allowing for coarser spatial discretization and, consequently, a larger time step. The stability conditions for both newly proposed schemes are derived and the numerical dispersion error is studied. In this work, the focus is on the nonuniform and higher-order spatial discretization, which is applied for one possible - but widely used - temporal discretization scheme. Still, it is applicable to other temporal discretizations $[1,7,12,13]$. The methods are validated using analytical solutions to a dynamical problem and their accuracy and efficiency are demonstrated.

In Section 2, the definition of the grid is given and the two update schemes are derived. The stability condition for both schemes is determined in Section 3. In Section 4, the numerical dispersion error is studied as a function of the propagation direction, the spatial discretization and the time step. The theoretical derivations are verified through numerical experimentation in Section 5. In Section 6, the results are summarized.

\section{Description of the proposed method}

The equation to be solved is the continuous time-dependent Schrödinger equation, given by

$$
\jmath \hbar \frac{\partial \psi}{\partial t}=-\frac{\hbar^{2}}{2 m^{*}} \nabla^{2} \psi+V \psi,
$$

where $\jmath$ is the imaginary unit, $\hbar$ the reduced Planck constant, $\psi$ the wave function, $m^{*}$ the particle's effective mass, $\nabla^{2}$ the Laplacian operator and $V$ the potential energy. To solve this equation, the wave function is discretized on a space-time structured grid.

\subsection{Spatial discretization}

The wave function is defined on the nodes of a 3-D nonuniform tensor product grid containing $n_{x} \times n_{y} \times n_{z}$ cells. The node $(i, j, k)$ of the grid corresponds to the position $\left(x_{i}, y_{j}, z_{k}\right)$ in real space. The value of the wave function at this position is denoted by $\left.\psi\right|_{i, j, k}$. The dual grid nodes are defined on the centroids of the primary grid. The spatial grid variables are defined in Fig. 1 for the $x$-direction.

The finite-difference approximation of the second-order derivative is obtained by solving for the coefficients $\left.c_{1}^{x}\right|_{i}$ and $\left.c_{-1}^{x}\right|_{i}$ of the Taylor expansion

$$
\left.\left.c_{1}^{x}\right|_{i} \psi\right|_{i+1, j, k}+\left.\left.c_{-1}^{x}\right|_{i} \psi\right|_{i-1, j, k}=\left.\sum_{m=0}^{\infty} \frac{1}{m !}\left(\left.c_{1}^{x}\right|_{i}\left(x_{i+1}-x_{i}\right)^{m}+\left.c_{-1}^{x}\right|_{i}\left(x_{i-1}-x_{i}\right)^{m}\right) \frac{\partial^{m} \psi}{\partial x^{m}}\right|_{i, j, k},
$$

such that the first-order derivative drops out. The resulting approximation is

$$
\left.\frac{\partial^{2} \psi}{\partial x^{2}}\right|_{i, j, k}=\frac{\left.\psi\right|_{i+1, j, k}-\left.\psi\right|_{i, j, k}}{\Delta x_{i} \Delta x_{i}^{\star}}-\frac{\left.\psi\right|_{i, j, k}-\left.\psi\right|_{i-1, j, k}}{\Delta x_{i}^{\star} \Delta x_{i-1}}+\mathcal{O}\left(\Delta x_{i}-\Delta x_{i-1}\right) .
$$


Hence, the first-order accurate approximation to the Laplacian, denoted $\mathfrak{L}_{L}$, is given by

$$
\begin{aligned}
\mathfrak{L}_{L}[\psi]_{i, j, k}= & \frac{\left.\psi\right|_{i+1, j, k}-\left.\psi\right|_{i, j, k}}{\Delta x_{i} \Delta x_{i}^{\star}}-\frac{\left.\psi\right|_{i, j, k}-\left.\psi\right|_{i-1, j, k}}{\Delta x_{i}^{\star} \Delta x_{i-1}} \\
& +\frac{\left.\psi\right|_{i, j+1, k}-\left.\psi\right|_{i, j, k}}{\Delta y_{j} \Delta y_{j}^{\star}}-\frac{\left.\psi\right|_{i, j, k}-\left.\psi\right|_{i, j-1, k}}{\Delta y_{j}^{\star} \Delta y_{j-1}} \\
& +\frac{\left.\psi\right|_{i, j, k+1}-\left.\psi\right|_{i, j, k}}{\Delta z_{k} \Delta z_{k}^{\star}}-\frac{\left.\psi\right|_{i, j, k}-\left.\psi\right|_{i, j, k-1}}{\Delta z_{k}^{\star} \Delta z_{k-1}} .
\end{aligned}
$$

For a uniform grid, this scheme is second-order accurate. Moreover, if a rather smoothly varying grid is used, the scheme will be close to second-order accurate. The subscript $L$, indicates the lower-order nature as to distinguish it from the higher-order scheme that will be proposed next.

The higher-order accurate spatial scheme is derived by including $\left.\psi\right|_{i+2, j, k},\left.\psi\right|_{i+1, j, k},\left.\psi\right|_{i-1, j, k}$ and $\left.\psi\right|_{i-2, j, k}$ in the Taylor expansion

$$
\begin{aligned}
& \left.\left.d_{2}^{x}\right|_{i} \psi\right|_{i+2, j, k}+\left.\left.d_{1}^{x}\right|_{i} \psi\right|_{i+1, j, k}+\left.\left.d_{-1}^{x}\right|_{i} \psi\right|_{i-1, j, k}+\left.\left.d_{-2}^{x}\right|_{i} \psi\right|_{i-2, j, k}= \\
& \left.\quad \sum_{m=0}^{\infty} \frac{1}{m !}\left(\left.d_{2}^{x}\right|_{i}\left(x_{i+2}-x_{i}\right)^{m}+\left.d_{1}^{x}\right|_{i}\left(x_{i+1}-x_{i}\right)^{m}+\left.d_{-1}^{x}\right|_{i}\left(x_{i-1}-x_{i}\right)^{m}+\left.d_{-2}^{x}\right|_{i}\left(x_{i-2}-x_{i}\right)^{m}\right) \frac{\partial^{m} \psi}{\partial x^{m}}\right|_{i, j, k}
\end{aligned}
$$

and requiring that the first-, third- and fourth-order derivative drop out. This yields the following set of equations

$$
\begin{aligned}
& \left.d_{2}^{x}\right|_{i}\left(x_{i+2}-x_{i}\right)+\left.d_{1}^{x}\right|_{i}\left(x_{i+1}-x_{i}\right)+\left.d_{-1}^{x}\right|_{i}\left(x_{i-1}-x_{i}\right)+\left.d_{-2}^{x}\right|_{i}\left(x_{i-2}-x_{i}\right)=0 \\
& \left.d_{2}^{x}\right|_{i}\left(x_{i+2}-x_{i}\right)^{3}+\left.d_{1}^{x}\right|_{i}\left(x_{i+1}-x_{i}\right)^{3}+\left.d_{-1}^{x}\right|_{i}\left(x_{i-1}-x_{i}\right)^{3}+\left.d_{-2}^{x}\right|_{i}\left(x_{i-2}-x_{i}\right)^{3}=0 \\
& \left.d_{2}^{x}\right|_{i}\left(x_{i+2}-x_{i}\right)^{4}+\left.d_{1}^{x}\right|_{i}\left(x_{i+1}-x_{i}\right)^{4}+\left.d_{-1}^{x}\right|_{i}\left(x_{i-1}-x_{i}\right)^{4}+\left.d_{-2}^{x}\right|_{i}\left(x_{i-2}-x_{i}\right)^{4}=0
\end{aligned}
$$

Also requiring that the coefficient for the second-order derivative equals 1 yields the equation

$$
\frac{1}{2}\left(\left.d_{2}^{x}\right|_{i}\left(x_{i+2}-x_{i}\right)^{2}+\left.d_{1}^{x}\right|_{i}\left(x_{i+1}-x_{i}\right)^{2}+\left.d_{-1}^{x}\right|_{i}\left(x_{i-1}-x_{i}\right)^{2}+\left.d_{-2}^{x}\right|_{i}\left(x_{i-2}-x_{i}\right)^{2}\right)=1 .
$$

The solution to (5)-(8) for the coefficients was obtained with a linear algebra package and is given by

$$
\begin{aligned}
\left.d_{-2}^{x}\right|_{i} & =\frac{2 \Delta x_{i+1}^{\star}\left(\Delta x_{i}-\Delta x_{i-1}\right)-\Delta x_{i} \Delta x_{i-1}}{2 \Delta x_{i-2} \Delta x_{i-1}^{\star}\left(2 \Delta x_{i-1}^{\star}+\Delta x_{i}\right)\left(\Delta x_{i-1}^{\star}+\Delta x_{i+1}^{\star}\right)}, \\
\left.d_{-1}^{x}\right|_{i} & =\frac{2\left(\Delta x_{i+1} \Delta x_{i-1}^{\star}+2 \Delta x_{i-1}^{\star} \Delta x_{i}-\Delta x_{i} \Delta x_{i+1}^{\star}\right)}{\Delta x_{i}^{\star} \Delta x_{i-1} \Delta x_{i-2}\left(2 \Delta x_{i}^{\star}+\Delta x_{i+1}\right)}, \\
\left.d_{1}^{x}\right|_{i} & =\frac{2\left(\Delta x_{i-2} \Delta x_{i+1}^{\star}+2 \Delta x_{i+1}^{\star} \Delta x_{i-1}-\Delta x_{i-1} \Delta x_{i-1}^{\star}\right)}{\Delta x_{i}^{\star} \Delta x_{i} \Delta x_{i+1}\left(2 \Delta x_{i}^{\star}+\Delta x_{i-2}\right)}, \\
\left.d_{2}^{x}\right|_{i} & =\frac{2 \Delta x_{i-1}^{\star}\left(\Delta x_{i-1}-\Delta x_{i}\right)-\Delta x_{i} \Delta x_{i-1}}{2 \Delta x_{i+1} \Delta x_{i+1}^{\star}\left(2 \Delta x_{i+1}^{\star}+\Delta x_{i-1}\right)\left(\Delta x_{i-1}^{\star}+\Delta x_{i+1}^{\star}\right)} .
\end{aligned}
$$

Rewriting (4) using (5)-(12) yields

$$
\begin{aligned}
\left.\frac{\partial^{2} \psi}{\partial x^{2}}\right|_{i, j, k}= & \left.d_{2}^{x}\right|_{i}\left(\left.\psi\right|_{i+2, j, k}-\left.\psi\right|_{i, j, k}\right)+\left.d_{1}^{x}\right|_{i}\left(\left.\psi\right|_{i+1, j, k}-\left.\psi\right|_{i, j, k}\right) \\
& +\left.d_{-1}^{x}\right|_{i}\left(\left.\psi\right|_{i-1, j, k}-\left.\psi\right|_{i, j, k}\right)+\left.d_{-2}^{x}\right|_{i}\left(\left.\psi\right|_{i-2, j, k}-\left.\psi\right|_{i, j, k}\right)+\mathcal{E}
\end{aligned}
$$


with the error term

$\mathcal{E}=\left.\sum_{m=5}^{\infty} \frac{2}{m !} \frac{\left.d_{2}^{x}\right|_{i}\left(\Delta x_{i}+\Delta x_{i+1}\right)^{m}+\left.d_{1}^{x}\right|_{i}\left(\Delta x_{i}\right)^{m}+\left.d_{-1}^{x}\right|_{i}\left(-\Delta x_{i-1}\right)^{m}+\left.d_{-2}^{x}\right|_{i}\left(-\Delta x_{i-2}-\Delta x_{i-1}\right)^{m}}{\left.d_{2}^{x}\right|_{i}\left(\Delta x_{i}+\Delta x_{i+1}\right)^{2}+\left.d_{1}^{x}\right|_{i}\left(\Delta x_{i}\right)^{2}+\left.d_{-1}^{x}\right|_{i}\left(\Delta x_{i-1}\right)^{2}+\left.d_{-2}^{x}\right|_{i}\left(\Delta x_{i-2}+\Delta x_{i-1}\right)^{2}} \frac{\partial^{m} \psi}{\partial x^{m}}\right|_{i, j, k}$.

To obtain the order of accuracy, the expressions for $\left.d_{2}^{x}\right|_{i},\left.d_{1}^{x}\right|_{i},\left.d_{-1}^{x}\right|_{i}$ and $\left.d_{-2}^{x}\right|_{i}$ are plugged into the $m=5$ term of the error. By simplifying the result with a linear algebra package and rewriting it as a function of the primary grid cells, it is seen that the second-order derivative is correct up to third-order

$$
\begin{aligned}
\mathcal{E}=\mathcal{O}\left(2 \Delta x_{i} \Delta x_{i-1}\right. & \left(\left(\Delta x_{i-2}+\Delta x_{i-1}\right)-\left(\Delta x_{i}+\Delta x_{i+1}\right)\right) \\
& \left.+\Delta x_{i-1} \Delta x_{i+1}\left(\Delta x_{i-2}+\Delta x_{i-1}\right)-\Delta x_{i} \Delta x_{i-2}\left(\Delta x_{i}+\Delta x_{i+1}\right)\right) .
\end{aligned}
$$

The third-order correct approximation of the Laplacian, denoted $\mathfrak{L}_{H}[\psi]_{i, j, k}$, is fourth-order accurate on uniform grids and is given by

$$
\begin{aligned}
\mathfrak{L}_{H}[\psi]_{i, j, k}= & \left.d_{2}^{x}\right|_{i}\left(\left.\psi\right|_{i+2, j, k}-\left.\psi\right|_{i, j, k}\right)+\left.d_{1}^{x}\right|_{i}\left(\left.\psi\right|_{i+1, j, k}-\left.\psi\right|_{i, j, k}\right) \\
& +\left.d_{-1}^{x}\right|_{i}\left(\left.\psi\right|_{i-1, j, k}-\left.\psi\right|_{i, j, k}\right)+\left.d_{-2}^{x}\right|_{i}\left(\left.\psi\right|_{i-2, j, k}-\left.\psi\right|_{i, j, k}\right) \\
& +\left.d_{2}^{y}\right|_{j}\left(\left.\psi\right|_{i, j+2, k}-\left.\psi\right|_{i, j, k}\right)+\left.d_{1}^{y}\right|_{j}\left(\left.\psi\right|_{i, j+1, k}-\left.\psi\right|_{i, j, k}\right) \\
& +\left.d_{-1}^{y}\right|_{j}\left(\left.\psi\right|_{i, j-1, k}-\left.\psi\right|_{i, j, k}\right)+\left.d_{-2}^{y}\right|_{j}\left(\left.\psi\right|_{i, j-2, k}-\left.\psi\right|_{i, j, k}\right) \\
& +\left.d_{2}^{z}\right|_{k}\left(\left.\psi\right|_{i, j, k+2}-\left.\psi\right|_{i, j, k}\right)+\left.d_{1}^{z}\right|_{k}\left(\left.\psi\right|_{i, j, k+1}-\left.\psi\right|_{i, j, k}\right) \\
& +\left.d_{-1}^{z}\right|_{k}\left(\left.\psi\right|_{i, j, k-1}-\left.\psi\right|_{i, j, k}\right)+\left.d_{-2}^{z}\right|_{k}\left(\left.\psi\right|_{i, j, k-2}-\left.\psi\right|_{i, j, k}\right) .
\end{aligned}
$$

\subsection{Temporal discretization}

A second-order accurate central difference is used for the temporal discretization [2]:

$$
\left.\frac{\partial \psi}{\partial t}\right|^{n}=\frac{\left.\psi\right|^{n+1}-\left.\psi\right|^{n-1}}{2 \Delta t}+\mathcal{O}\left(\Delta t^{2}\right)
$$

where $n$ denotes the temporal index.

Combining the spatial and temporal discretizations yields following update scheme

$$
\left.\psi\right|_{i, j, k} ^{n+1}=\left.\psi\right|_{i, j, k} ^{n-1}+\jmath \frac{\hbar \Delta t}{m^{*}} \mathfrak{L}[\psi]_{i, j, k}^{n}-\left.\left.\jmath \frac{2 \Delta t}{\hbar} V\right|_{i, j, k} \psi\right|_{i, j, k} ^{n}
$$

where $\mathfrak{L}$ is either $\mathfrak{L}_{L}$ or $\mathfrak{L}_{H}$, for the lower- or higher-order scheme, respectively. This update scheme (18) naturally leads to two independent leapfrog schemes, as the real and imaginary parts at even and odd time steps, respectively, are decoupled from the real and imaginary parts at odd and even time steps, respectively (Fig. 2). With $\psi_{\operatorname{Re}}$ and $\psi_{\operatorname{Im}}$ the real and imaginary parts of the wave function, the update equations are given by

$$
\begin{aligned}
\left.\psi_{\operatorname{Re}}\right|_{i, j, k} ^{2 n} & =\left.\psi_{\operatorname{Re}}\right|_{i, j, k} ^{2 n-2}-\frac{\hbar \Delta t}{m^{*}} \mathfrak{L}\left[\psi_{\operatorname{Im}}\right]_{i, j, k}^{2 n-1}+\left.\left.\frac{2 \Delta t}{\hbar} V\right|_{i, j, k} \psi_{\operatorname{Im}}\right|_{i, j, k} ^{2 n-1}, \\
\left.\psi_{\operatorname{Im}}\right|_{i, j, k} ^{2 n+1} & =\left.\psi_{\operatorname{Im}}\right|_{i, j, k} ^{2 n-1}+\frac{\hbar \Delta t}{m^{*}} \mathfrak{L}\left[\psi_{\operatorname{Re}}\right]_{i, j, k}^{2 n}-\left.\left.\frac{2 \Delta t}{\hbar} V\right|_{i, j, k} \psi_{\operatorname{Re}}\right|_{i, j, k} ^{2 n},
\end{aligned}
$$

and

$$
\begin{aligned}
& \left.\psi_{\operatorname{Re}}\right|_{i, j, k} ^{2 n+1}=\left.\psi_{\operatorname{Re}}\right|_{i, j, k} ^{2 n-1}-\frac{\hbar \Delta t}{m^{*}} \mathfrak{L}\left[\psi_{\operatorname{Im}}\right]_{i, j, k}^{2 n}+\left.\left.\frac{2 \Delta t}{\hbar} V\right|_{i, j, k} \psi_{\operatorname{Im}}\right|_{i, j, k} ^{2 n}, \\
& \left.\psi_{\operatorname{Im}}\right|_{i, j, k} ^{2 n+2}=\left.\psi_{\operatorname{Im}}\right|_{i, j, k} ^{2 n}+\frac{\hbar \Delta t}{m^{*}} \mathfrak{L}\left[\psi_{\operatorname{Re}}\right]_{i, j, k}^{2 n+1}-\left.\left.\frac{2 \Delta t}{\hbar} V\right|_{i, j, k} \psi_{\operatorname{Re}}\right|_{i, j, k} ^{2 n+1} .
\end{aligned}
$$



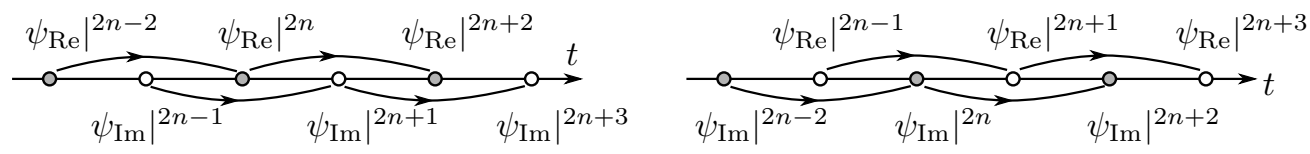

Figure 2: The two intertwined leapfrog schemes. The left scheme, with the real part $\psi_{\text {Re }}$ at even and the imaginary part $\psi_{\text {Im }}$ at odd time steps, is decoupled from the right scheme, with the real part at odd and imaginary part at even time steps. In the numerical implementation, it is twice as efficient to solve only one of both decoupled schemes.

For notational simplicity, it is easier to write the wave function as the complex scalar equation, as in (18). However, for computational efficiency, the real and imaginary parts should be split and only one of both leapfrog schemes should be computed, either (19)-(20) or (21)-(22), as was also proposed in [3]. Even though only (18) will be considered in the remainder of this paper, the results are valid for all schemes.

The proposed nonuniform and higher-order spatial discretization can be combined with other temporal schemes such as the Crank-Nicolson method [1] or the Alternating-Direction Implicit method [12]. It is also possible to combine it with higher-order temporal schemes, e.g. the generalized FDTD method [7] or a higher-order symplectic scheme [13]. However, the application of these schemes and their stability analysis is beyond the scope of this work.

The proposed schemes can incorporate a perfectly matched layer (PML) [14] to allow a particle to leave the computational domain. The PML technique for the Schrödinger equation, where a coordinate stretching is performed into the complex plane [15-18], is applied to (18) both for the lower-order and higher-order accurate scheme. The cells have to be stretched as

$$
\begin{aligned}
\Delta x_{i, \mathrm{PML}} & =\Delta x_{i}\left(1+\left.R \sigma\right|_{i+1 / 2}\right), \\
\Delta x_{i, \mathrm{PML}}^{\star} & =\Delta x_{i}^{\star}\left(1+\left.R \sigma\right|_{i}\right),
\end{aligned}
$$

where $R$ is a complex number and $\sigma$ the absorption function. The absorption function increases as a function of the depth inside the PML and $R$ determines the ratio of real versus imaginary stretch [19]. If the complex cell sizes are used directly in the Laplacian operator $\mathfrak{L}[\psi]_{i, j, k}$, it becomes a complex operator. This mixes the two independent leapfrog schemes (19)-(20) and (21)-(22) and renders the scheme unstable, as will be discussed in Section 3. To remedy this, the approach described in [17] is applied, resulting in

$$
\left.\psi\right|_{i, j, k} ^{n+1}=\left.\psi\right|_{i, j, k} ^{n-1}-\frac{\hbar \Delta t}{m^{*}} \mathfrak{L}_{\operatorname{Im}}[\psi]_{i, j, k}^{n-1}+\jmath \frac{\hbar \Delta t}{m^{*}} \mathfrak{L}_{\operatorname{Re}}[\psi]_{i, j, k}^{n}-\left.\left.\jmath \frac{2 \Delta t}{\hbar} V\right|_{i, j, k} \psi\right|_{i, j, k} ^{n},
$$

where $\mathfrak{L}_{\operatorname{Re}}[\psi]_{i, j, k}$ and $\mathfrak{L}_{\operatorname{Im}}[\psi]_{i, j, k}$ are the real and imaginary parts of $\mathfrak{L}[\psi]_{i, j, k}$, respectively.

\section{Stability}

The stability of a time-stepping scheme is of utmost importance. For uniform grids, a condition on the time step is generally derived by a Von Neumann analysis, where the grid is implicitly assumed infinite and the potential constant $[7,8,13,20,21]$. This approach was further verified for the Schrödinger equation in [6], where a more rigorous criterion was determined by explicitly allowing the potential to vary. For nonuniform grids, however, the stability condition cannot be derived with this method. Instead, we adopt discrete-time stability analysis for matrices to derive a rigorous stability condition [22]. Additionally, this analysis offers a deeper insight into the true time step stability bound, which is dictated by the eigenvalues of the iteration matrix, even in the limit of uniform grids.

The update equation (18) is written in matrix form as

$$
\nu^{n}=\mathbf{A} \nu^{n-1}
$$


with

$$
\nu^{n}=\left[\begin{array}{c}
\psi^{n} \\
\psi^{n-1}
\end{array}\right] \text { and } \mathbf{A}=\left[\begin{array}{cc}
-\jmath \frac{2 \Delta t}{\hbar} \mathbf{H} & \mathbf{I} \\
\mathbf{I} & \mathbf{0}
\end{array}\right]
$$

where $\psi^{n}$ is the row-major vectorization of the wave function at time index $n$. The Hamiltonian matrix is defined as

$$
\mathbf{H} \triangleq-\frac{\hbar^{2}}{2 m^{*}} \mathbf{L}+\mathbf{V}
$$

with $\mathbf{L}$ the discretized Laplacian operator, either for the lower-order or higher-order scheme. $\mathbf{V}$ is the diagonal matrix containing the potential energy.

Theorem 1. The FDTD scheme (26) is stable if and only if the eigenvalues of $\mathbf{H}$ are real and the time step satisfies the relation

$$
\Delta t \leq \frac{\hbar}{\rho(\mathbf{H})},
$$

where $\rho(\cdot)$ is the spectral radius.

Proof. To guarantee stability, the eigenvalues $z$ of the iteration matrix $\mathbf{A}$ cannot lie outside the unit circle, i.e. $|z| \leq 1[22-24]$. Since $\operatorname{det}(z \mathbf{I}-\mathbf{A})=\operatorname{det}\left(z^{2} \mathbf{I}+z\left(\jmath \frac{2 \Delta t}{\hbar} \mathbf{H}\right)-\mathbf{I}\right)[22]$, the eigenvalues $z$ are related to the eigenvalues $w$ of $\mathbf{H}$ through:

$$
z^{2}+z\left(\jmath \frac{2 \Delta t}{\hbar} w\right)-1=0
$$

The eigenvalues $z$ lie in the closed unit circle if and only if the following two conditions are simultaneously satisfied:

$$
\left|-\jmath \frac{\Delta t}{\hbar} w+\sqrt{1-\left(\frac{\Delta t}{\hbar} w\right)^{2}}\right| \leq 1
$$

and

$$
\left|-\jmath \frac{\Delta t}{\hbar} w-\sqrt{1-\left(\frac{\Delta t}{\hbar} w\right)^{2}}\right| \leq 1 .
$$

For this to be true, the eigenvalues $w$ of $\mathbf{H}$ have to be real and:

$$
\Delta t \leq \frac{\hbar}{\max (|w|)},
$$

where by definition $\rho(\mathbf{H})=\max (|w|)$ is the spectral radius of $\mathbf{H}$. Provided that this is true, the eigenvalues $z$ will all lie on the unit circle. For complex values of $w$ or for $\Delta t$ not satisfying (33), either (31) or (32) has a norm greater than 1 , yielding an unstable scheme.

This stability criterion is very general as it is valid for every possible spatial scheme that uses the collocated scheme (18) or the equivalent staggered in time schemes (19)-(20) or (21)-(22). For example, it would be possible to apply Theorem 1 to a Hamiltonian matrix with a Laplacian $\mathbf{L}$ constructed using secondorder forward or backward differences on a uniform grid or using the nonuniform grid mapping presented in [11]. In fact, the time step used in the latter was derived based on a uniform grid mapping. Moreover, Theorem 1 is valid for different kinds of boundary conditions.

Theorem 2. The eigenvalues of $\mathbf{H}_{L}$ of the nonuniform lower-order scheme, using Dirichlet boundary conditions, are real. 
Proof. The Laplacian for the lower-order scheme $\mathbf{L}_{L}$, can be decomposed as:

$$
\mathbf{L}_{L}=\mathbf{L}_{L x} \oplus \mathbf{L}_{L y} \oplus \mathbf{L}_{L z}=-\mathbf{D}_{x}^{\star} \mathbf{D}_{x} \oplus \mathbf{D}_{y}^{\star} \mathbf{D}_{y} \oplus \mathbf{D}_{z}^{\star} \mathbf{D}_{z}
$$

where $\oplus$ is the Kronecker sum and the discrete derivatives are defined as:

$$
\begin{aligned}
& \mathbf{D}_{u}^{\star}=\boldsymbol{\delta}_{u}^{\star} \mathbf{F}_{u}^{T}=\operatorname{Diag}\left(\Delta u_{1}^{\star}, \ldots, \Delta u_{n_{u}-1}^{\star}\right)^{-1}\left[\begin{array}{ccccc}
-1 & 1 & & & \\
& -1 & 1 & & \\
& \ddots & \ddots & \\
& & -1 & 1
\end{array}\right]_{\left(n_{u}-1\right) \times n_{u}}, \\
& \mathbf{D}_{u}=\boldsymbol{\delta}_{u} \mathbf{F}_{u}=\operatorname{Diag}\left(\Delta u_{0}, \ldots, \Delta u_{n_{u}-1}\right)^{-1}\left[\begin{array}{cccc}
-1 & & & \\
1 & -1 & & \\
& \ddots & \ddots & \\
& & 1 & -1 \\
& & & 1
\end{array}\right]_{n_{u} \times\left(n_{u}-1\right)},
\end{aligned}
$$

for $u \in\{x, y, z\}$. For a grid consisting of $n_{x} \times n_{y} \times n_{z}$ cells and using Dirichlet boundary conditions, the wave function is different from zero only on $\left(n_{x}-1\right) \times\left(n_{y}-1\right) \times\left(n_{z}-1\right)$ nodes, which is accounted for in $\mathbf{F}_{x}, \mathbf{F}_{y}$ and $\mathbf{F}_{z}$. With a similarity transformation, $\mathbf{H}_{L}$ is brought into a different form, without changing the eigenvalues:

$$
\begin{aligned}
\tilde{\mathbf{H}}_{L} & =\mathbf{Q}^{-\frac{1}{2}} \mathbf{H}_{L} \mathbf{Q}^{\frac{1}{2}} \\
& =-\frac{\hbar^{2}}{2 m^{*}} \mathbf{Q}^{-\frac{1}{2}} \mathbf{L}_{L} \mathbf{Q}^{\frac{1}{2}}+\mathbf{V} \\
& =-\frac{\hbar^{2}}{2 m^{*}} \tilde{\mathbf{L}}_{L}+\mathbf{V}
\end{aligned}
$$

where the diagonal transformation matrix $\mathbf{Q}$ is composed of the dual grid steps as follows:

$$
\mathbf{Q} \triangleq \boldsymbol{\delta}_{x}^{\star} \otimes \boldsymbol{\delta}_{y}^{\star} \otimes \boldsymbol{\delta}_{z}^{\star}
$$

where $\otimes$ is the Kronecker product. The Laplacian $\mathbf{L}_{L}$ is expanded as:

$$
\mathbf{L}_{L}=-\left(\mathbf{D}_{x}^{\star} \mathbf{D}_{x} \otimes \mathbf{I}_{n_{y}-1} \otimes \mathbf{I}_{n_{z}-1}\right)-\left(\mathbf{I}_{n_{x}-1} \otimes \mathbf{D}_{y}^{\star} \mathbf{D}_{y} \otimes \mathbf{I}_{n_{z}-1}\right)-\left(\mathbf{I}_{n_{x}-1} \otimes \mathbf{I}_{n_{y}-1} \otimes \mathbf{D}_{z}^{\star} \mathbf{D}_{z}\right) .
$$

Hence, the Laplacian $\mathbf{L}_{L}$ transforms as:

$$
\tilde{\mathbf{L}}_{L}=-\left(\left(\boldsymbol{\delta}_{x}^{\star}\right)^{-1 / 2} \mathbf{D}_{x}^{\star} \mathbf{D}_{x}\left(\boldsymbol{\delta}_{x}^{\star}\right)^{1 / 2}\right) \oplus\left(\left(\boldsymbol{\delta}_{y}^{\star}\right)^{-1 / 2} \mathbf{D}_{y}^{\star} \mathbf{D}_{y}\left(\boldsymbol{\delta}_{y}^{\star}\right)^{1 / 2}\right) \oplus\left(\left(\boldsymbol{\delta}_{z}^{\star}\right)^{-1 / 2} \mathbf{D}_{z}^{\star} \mathbf{D}_{z}\left(\boldsymbol{\delta}_{z}^{\star}\right)^{1 / 2}\right)
$$

where we have used the mixed-product property for the Kronecker product $(\mathbf{A} \otimes \mathbf{B})(\mathbf{C} \otimes \mathbf{D})=(\mathbf{A C}) \otimes(\mathbf{B D})$ [22]. The matrix $\tilde{\mathbf{L}}_{L}$ is real symmetric since it is readily seen that

$$
\left(\boldsymbol{\delta}_{u}^{\star}\right)^{-1 / 2} \mathbf{D}_{u}^{\star} \mathbf{D}_{u}\left(\boldsymbol{\delta}_{u}^{\star}\right)^{1 / 2}=\left(\boldsymbol{\delta}_{u}^{\star}\right)^{1 / 2} \mathbf{F}_{u}^{T} \boldsymbol{\delta}_{u} \mathbf{F}_{u}\left(\boldsymbol{\delta}_{u}^{\star}\right)^{1 / 2}, \quad u \in\{x, y, z\}
$$

is real symmetric. Consequently, $\mathbf{L}_{L}$ and thus also $\mathbf{H}_{L}$ has only real eigenvalues.

The proof can be repeated for periodic boundary conditions with minor changes. An extra column has to be added to $\mathbf{F}_{u}$ as follows:

$$
\mathbf{F}_{u}=\left[\begin{array}{ccccc}
-1 & & & & 1 \\
1 & -1 & & & \\
& \ddots & \ddots & & \\
& & 1 & -1 & \\
& & & 1 & -1
\end{array}\right]_{n_{u} \times n_{u}}
$$


and $\boldsymbol{\delta}_{u}^{\star}$ becomes $\boldsymbol{\delta}_{u}^{\star}=\operatorname{Diag}\left(\Delta u_{0}^{\star}, \ldots, \Delta u_{n_{u}-1}^{\star}\right)^{-1}$. The remainder of the proof remains unchanged.

The proof also shows why the direct implementation of a PML with complex cell sizes would lead to an unstable scheme. Then, the matrix $\mathbf{L}$ can be shown to be similar to a complex symmetric matrix instead of a real symmetric or complex hermitian matrix, which does not necessarily yield real eigenvalues. Therefore, we adopt the alternative PML implementation (25) from [17] and numerical experiments show that this method is indeed stable.

The Laplacian $\mathbf{L}_{H}$ of the higher-order scheme can also be decomposed as the Kronecker sum $\mathbf{L}_{H}=$ $\mathbf{L}_{H x} \oplus \mathbf{L}_{H y} \oplus \mathbf{L}_{H z}$ with $\mathbf{L}_{H x}, \mathbf{L}_{H y}$ and $\mathbf{L}_{H z}$ the discretized third-order accurate second-order derivatives in the $x$-, $y$ - and $z$-directions, respectively. However, it is not possible to decompose this further and prove the similarity to a real symmetric matrix of $\mathbf{H}_{H}$ in a straightforward manner. In the case of a uniform grid, $\mathbf{H}_{H}$ is a real and symmetric matrix as $\mathbf{L}_{H x}, \mathbf{L}_{H y}$ and $\mathbf{L}_{H z}$ are real symmetric, which implies that the eigenvalues are real. Hence, the inequality (29) will guarantee stability. In the next section, however, we will assume that $\mathbf{H}_{H}$ has only real eigenvalues, even in the nonuniform case, which is supported by numerical calculations provided in Section 5.

\subsection{A Courant-like stability criterion}

Calculating the eigenvalues of $\mathbf{H}$ numerically is computationally expensive for large grids. In most practical cases, the time step is based on an easily calculable formula, such as the Courant-Friedrichs-Lewy condition, which makes a (slight) underestimate of the stability limit [25-27]. In order to obtain a similar condition, an estimate of the spectral radius of $\mathbf{H}$ is made next.

The spectral radius is bounded by any induced norm, e.g. the infinity norm [28]:

$$
\rho(\mathbf{H}) \leq\|\mathbf{H}\|_{\infty}=\max _{p} \sum_{r}\left|H_{p r}\right| .
$$

To derive a Courant-like stability criterion for the lower-order scheme, the structure of the lower-order Hamiltonian matrix $\mathbf{H}_{L}$ has to be considered. Row $\left.m=k+\left(n_{z}-1\right)\left(j-1+\left(n_{y}-1\right)(i-1)\right)\right)$ with $m \in$ $\left\{1, \ldots,\left(n_{x}-1\right)\left(n_{y}-1\right)\left(n_{z}-1\right)\right\}, i \in\left\{1, \ldots, n_{x}-1\right\}, j \in\left\{1, \ldots, n_{y}-1\right\}$, and $k \in\left\{1, \ldots, n_{z}-1\right\}$ of the Hamiltonian matrix looks like

$$
\begin{aligned}
& {\left[\ldots,-\left.\frac{\hbar^{2}}{2 m^{*}} c_{-1}^{x}\right|_{i}, \ldots,-\left.\frac{\hbar^{2}}{2 m^{*}} c_{-1}^{y}\right|_{j}, \ldots,-\left.\frac{\hbar^{2}}{2 m^{*}} c_{-1}^{z}\right|_{k},\right.} \\
& -\frac{\hbar^{2}}{2 m^{*}}\left(-\left.c_{-1}^{x}\right|_{i}-\left.c_{1}^{x}\right|_{i}-\left.c_{-1}^{y}\right|_{j}-\left.c_{1}^{y}\right|_{j}-\left.c_{-1}^{z}\right|_{k}-\left.c_{1}^{z}\right|_{k}\right)+\left.V\right|_{i, j, k}, \\
& \left.-\left.\frac{\hbar^{2}}{2 m^{*}} c_{1}^{z}\right|_{k}, \ldots,-\left.\frac{\hbar^{2}}{2 m^{*}} c_{1}^{y}\right|_{j}, \ldots,-\left.\frac{\hbar^{2}}{2 m^{*}} c_{1}^{x}\right|_{i}, \ldots\right]
\end{aligned}
$$

where the "..." represent an appropriate number of zeros. The column indexes $n$ of the nonzero elements are in order: $n=m-\left(n_{z}-1\right)\left(n_{y}-1\right), n=m-\left(n_{z}-1\right), n=m-1, n=m, n=m+1, n=m+\left(n_{z}-1\right)$ and $n=m+\left(n_{z}-1\right)\left(n_{y}-1\right)$.

The row $m$ for which the sum of the absolute values of these elements is maximum, yields the infinity norm:

$$
\left\|\mathbf{H}_{L}\right\|_{\infty}=\max _{i, j, k}\left(\left[\left|\frac{\hbar^{2}}{2 m^{*}}\left(\frac{1}{\Lambda_{i, j, k}}\right)^{2}+V\right|_{i, j, k} \mid+\frac{\hbar^{2}}{2 m^{*}}\left(\frac{1}{\Lambda_{i, j, k}}\right)^{2}\right]\right),
$$

where $\Lambda_{i, j, k}$ is defined by

$$
\left(\frac{1}{\Lambda_{i, j, k}}\right)^{2} \triangleq \frac{1}{\Delta x_{i-1} \Delta x_{i}^{\star}}+\frac{1}{\Delta x_{i}^{\star} \Delta x_{i}}+\frac{1}{\Delta y_{j-1} \Delta y_{j}^{\star}}+\frac{1}{\Delta y_{j}^{\star} \Delta y_{j}}+\frac{1}{\Delta z_{k-1} \Delta z_{k}^{\star}}+\frac{1}{\Delta z_{k}^{\star} \Delta z_{k}} .
$$


As such, the Courant-like stability criterion for the lower-order scheme is

$$
\left.\Delta t_{L} \leq \min _{i, j, k}\left(|| \frac{\hbar}{2 m^{*}}\left(\frac{1}{\Lambda_{i, j, k}}\right)^{2}+\left.\frac{1}{\hbar} V\right|_{i, j, k} \mid+\frac{\hbar}{2 m^{*}}\left(\frac{1}{\Lambda_{i, j, k}}\right)^{2}\right]^{-1}\right) .
$$

A similar reasoning leads to the Courant-like stability criterion for the higher-order scheme:

$$
\begin{aligned}
& \Delta t_{H} \leq \min _{i, j, k}\left(\left[\left|\frac{\hbar}{2 m^{*}}\left(\left.\sum_{(u, l) \in\{(x, i),(y, j),(z, k)\}} d_{-2}^{u}\right|_{l}+\left.d_{-1}^{u}\right|_{l}+\left.d_{1}^{u}\right|_{l}+\left.d_{2}^{u}\right|_{l}\right)+\frac{1}{\hbar} V\right|_{i, j, k} \mid\right.\right. \\
& \left.\left.+\frac{\hbar}{2 m^{*}}\left(\left.\left.\left.\sum_{(u, l) \in\{(x, i),(y, j),(z, k)\}}\left|d_{-2}^{u}\right|_{l}|+| d_{-1}^{u}\right|_{l}|+| d_{1}^{u}\right|_{l}|+| d_{2}^{u}\right|_{l} \mid\right)\right]^{-1}\right) .
\end{aligned}
$$

On uniform grids, the Hamiltonian matrix $\mathbf{H}$ is symmetric and, hence, all its eigenvalues are real. In this case, the stability conditions simplify to:

$$
\begin{aligned}
& \Delta t_{L} \leq \min _{i, j, k} \frac{1}{\left|\frac{\hbar}{m^{*}}\left(\frac{1}{\Delta x^{2}}+\frac{1}{\Delta y^{2}}+\frac{1}{\Delta z^{2}}\right)+\frac{1}{\hbar} V\right|_{i, j, k} \mid+\frac{\hbar}{m^{*}}\left(\frac{1}{\Delta x^{2}}+\frac{1}{\Delta y^{2}}+\frac{1}{\Delta z^{2}}\right)}, \\
& \Delta t_{H} \leq \min _{i, j, k} \frac{1}{\left|\frac{5 \hbar}{4 m^{*}}\left(\frac{1}{\Delta x^{2}}+\frac{1}{\Delta y^{2}}+\frac{1}{\Delta z^{2}}\right)+\frac{1}{\hbar} V\right|_{i, j, k} \mid+\frac{17 \hbar}{12 m^{*}}\left(\frac{1}{\Delta x^{2}}+\frac{1}{\Delta y^{2}}+\frac{1}{\Delta z^{2}}\right)},
\end{aligned}
$$

for the lower- and higher-order scheme, respectively. On this uniform grid, these schemes are second- and fourth-order accurate, respectively.

In many practical cases the potential satisfies the relation

$$
\left.V\right|_{i, j, k} \geq-\frac{\hbar^{2}}{m^{*}}\left(\frac{1}{\Delta x^{2}}+\frac{1}{\Delta y^{2}}+\frac{1}{\Delta z^{2}}\right),
$$

simplifying the time steps to:

$$
\begin{aligned}
\Delta t_{L} & \leq \frac{1}{\frac{2 \hbar}{m^{*}}\left(\frac{1}{\Delta x^{2}}+\frac{1}{\Delta y^{2}}+\frac{1}{\Delta z^{2}}\right)+\left.\frac{1}{\hbar} V\right|_{\max }} \\
\Delta t_{H} & \leq \frac{1}{\frac{8 \hbar}{3 m^{*}}\left(\frac{1}{\Delta x^{2}}+\frac{1}{\Delta y^{2}}+\frac{1}{\Delta z^{2}}\right)+\left.\frac{1}{\hbar} V\right|_{\max }}
\end{aligned}
$$

which is consistent with results in $[3,6,7,13,29]$ when using only one of the two independent leapfrog schemes in Fig. 2.

From (46)-(52) it is clear that the scheme can be tuned to achieve a trade-off between efficiency (large time step) and accuracy (small spatial step). For example, (46) states that the grid can be made coarser (i.e. larger $\left.\Lambda_{i, j, k}\right)$ at locations where $\left.V\right|_{i, j, k}$ is larger. This property is exploited and demonstrated in Section 5 .

The calculation method is further explained in [30], where a script is provided able of calculating the time step for a given grid and potential. This script is also used later in this paper.

\section{Numerical dispersion error}

In this section, the numerical dispersion error is derived and analyzed for both new schemes. The dispersion error is characterized by the difference between the physical propagation of a plane wave and 
the one calculated by the FDTD algorithm. To derive this error, the grid is assumed uniform and infinite with a constant potential $V$, such that the following plane wave solution can be substituted into the update equations:

$$
\left.\psi\right|_{i, j, k} ^{n}=e^{-\jmath\left(\tilde{\omega} n \Delta t-k_{x} i \Delta x-k_{y} j \Delta y-k_{z} k \Delta z\right)},
$$

where $\tilde{\omega}$ is the numerical angular frequency, and $k_{x}, k_{y}$, and $k_{z}$, are the $x-, y$-, $z$-components of the wave vector $\mathbf{k}$, respectively. This plane wave corresponds to the solution of the Schrödinger equation that is enforced on the space-time discretized grid. Substitution of this plane wave into the lower-order scheme and multiplication by $e^{j\left(\tilde{\omega} n \Delta t-k_{x} i \Delta x-k_{y} j \Delta y-k_{z} k \Delta z\right)}$ yields

$$
\begin{aligned}
& \frac{1}{2 \Delta t}\left(e^{-\jmath \tilde{\omega} \Delta t}-e^{\jmath \tilde{\omega} \Delta t}\right)= \\
& \quad \jmath \frac{\hbar}{2 m^{*}}\left[\frac{e^{\jmath k_{x} \Delta x}-2+e^{-\jmath k_{x} \Delta x}}{\Delta x^{2}}+\frac{e^{\jmath k_{y} \Delta y}-2+e^{-\jmath k_{y} \Delta y}}{\Delta y^{2}}+\frac{e^{\jmath k_{z} \Delta z}-2+e^{-\jmath k_{z} \Delta z}}{\Delta z^{2}}\right]-\jmath \frac{V}{\hbar} .
\end{aligned}
$$

This simplifies to the dispersion relation

$$
\frac{1}{\Delta t} \sin (\tilde{\omega} \Delta t)=\frac{\hbar}{2 m^{*}}\left(\frac{4}{\Delta x^{2}} \sin ^{2}\left(\frac{k_{x} \Delta x}{2}\right)+\frac{4}{\Delta y^{2}} \sin ^{2}\left(\frac{k_{y} \Delta y}{2}\right)+\frac{4}{\Delta z^{2}} \sin ^{2}\left(\frac{k_{z} \Delta z}{2}\right)\right)+\frac{V}{\hbar} .
$$

Substitution into the higher-order scheme multiplication by $e^{\jmath\left(\tilde{\omega} n \Delta t-k_{x} i \Delta x-k_{y} j \Delta y-k_{z} k \Delta z\right)}$ yields

$$
\begin{aligned}
\frac{1}{2 \Delta t}\left(e^{-\jmath \tilde{\omega} \Delta t}-e^{\jmath \tilde{\omega} \Delta t}\right)=\jmath \frac{\hbar}{2 m^{*}} & {\left[\frac{1}{12 \Delta x^{2}}\left(-e^{\jmath 2 k_{x} \Delta x}-e^{-\jmath 2 k_{x} \Delta x}+16 e^{\jmath k_{x} \Delta x}+16 e^{-\jmath k_{x} \Delta x}-30\right)\right.} \\
& +\frac{1}{12 \Delta y^{2}}\left(-e^{\jmath 2 k_{y} \Delta y}-e^{-\jmath 2 k_{y} \Delta y}+16 e^{\jmath k_{y} \Delta y}+16 e^{-\jmath k_{y} \Delta y}-30\right) \\
& \left.+\frac{1}{12 \Delta z^{2}}\left(-e^{\jmath 2 k_{z} \Delta z}-e^{-\jmath 2 k_{z} \Delta z}+16 e^{\jmath k_{z} \Delta z}+16 e^{-\jmath k_{z} \Delta z}-30\right)\right]-\jmath \frac{V}{\hbar} .
\end{aligned}
$$

This simplifies to the dispersion relation for the higher-order scheme

$$
\begin{aligned}
\frac{1}{\Delta t} \sin (\tilde{\omega} \Delta t)=\frac{\hbar}{2 m^{*}} & \left(\frac{1}{3 \Delta x^{2}}\left(16 \sin ^{2}\left(\frac{k_{x} \Delta x}{2}\right)-\sin ^{2}\left(k_{x} \Delta x\right)\right)\right. \\
& +\frac{1}{3 \Delta y^{2}}\left(16 \sin ^{2}\left(\frac{k_{y} \Delta y}{2}\right)-\sin ^{2}\left(k_{y} \Delta y\right)\right) \\
& \left.+\frac{1}{3 \Delta z^{2}}\left(16 \sin ^{2}\left(\frac{k_{z} \Delta z}{2}\right)-\sin ^{2}\left(k_{z} \Delta z\right)\right)\right)+\frac{V}{\hbar} .
\end{aligned}
$$

In the limit of $\tilde{\omega} \Delta t \rightarrow 0$ and $\|\mathbf{k}\| \Delta \rightarrow 0$, both (55) and (57) reduce to the analytical relation

$$
\hbar \omega=\frac{\hbar^{2}\|\mathbf{k}\|^{2}}{2 m^{*}}+V .
$$

An error measure is defined as

$$
\mathcal{E}=\frac{\omega-\tilde{\omega}}{\omega},
$$

which measures the relative difference between the numerical and the exact energy. We investigate the directional dependence of the error $\mathcal{E}$ by writing the wave vector in spherical coordinates and solving (55) and $(57)$ for $\tilde{\omega}$. First, we study the influence of the ratio of kinetic and potential energy. In Fig. 3, the dispersion error for a free particle, i.e. $V=0$, is given for a discretization of 20 samples per wavelength $\lambda=2 \pi /\|\mathbf{k}\|$ with time steps chosen at the upper bound of (51) and (52) for the lower- and higher-order scheme, respectively. The maximum error for the lower-order scheme is approximately 80 times larger compared to the higher-order scheme. For both schemes, the body diagonals are the directions of least error, 


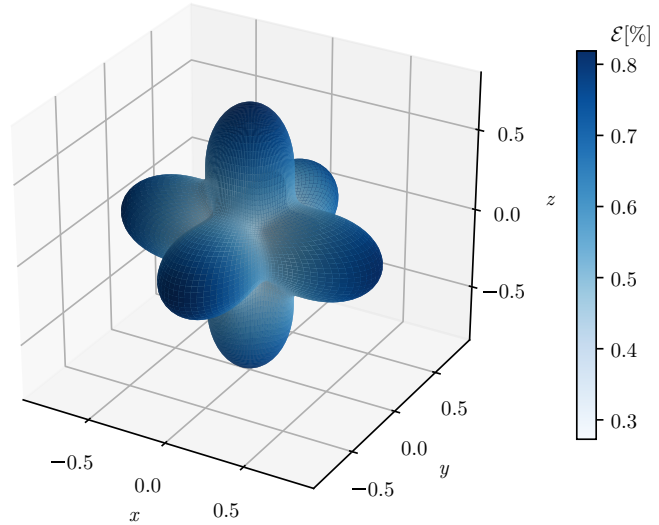

(a) Lower-order scheme.

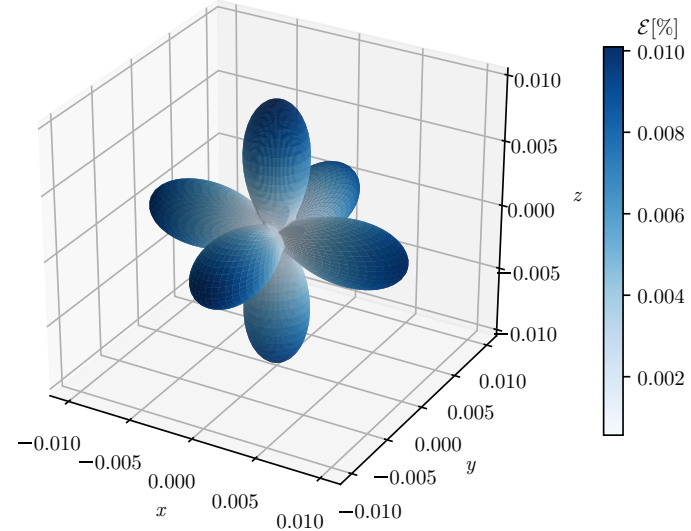

(b) Higher-order scheme.

Figure 3: Directional dependence of the dispersion error $\mathcal{E}(59)$ of a free particle $(V=0)$ for both schemes. A discretization of 20 samples per wavelength is adopted and the time step is chosen at the upper bound of (51) and (52). The maximum error for the lower-order scheme is approximately 80 times larger compared to the higher-order scheme.

but for neither scheme does the error reduce to zero. The higher-order scheme exhibits more anisotropic behavior, but this is largely compensated by the overall decrease in error. In Fig. 4, the potential is set equal to the kinetic energy whereas the other variables remain unchanged. The maximal error of the lower-order scheme is more than 30 times larger compared to the higher-order scheme. The directional dependence is more intricate as the numerical angular frequency $\tilde{\omega}$ can now also run ahead of the analytical angular frequency $\omega$, yielding a negative error $\mathcal{E}$. The directional dependence will change when varying the potential energy. In Fig. 5, the numerical dispersion error is plotted along the body diagonal for a free particle. The higher-order scheme is seen to perform orders of magnitude better compared to the lower-order scheme. From Fig. 5a, it is observed that the error drops faster upon reducing the spatial step. From Fig. 5b, however, it is seen that decreasing the time step does not yield better results. For computational efficiency and accuracy, it is beneficial to choose the time step as high as possible, within the stability range.

\section{Numerical Validation}

\subsection{Eigenvalues of $\mathbf{H}$ and $\mathbf{A}$}

In this section, the eigenvalues of $\mathbf{H}$ and $\mathbf{A}$ are studied as a function of the cell sizes, the chosen time step and the potential energy. Visualizing how the eigenvalues change, shows the origin of instabilities. The contribution of an eigenvector corresponding to eigenvalues outside the unit circle will grow at every time step, resulting in an unstable scheme. Even if the contributions of all unstable eigenvectors are zero at the start of the simulation, it is possible that the scheme will become unstable due to numerical rounding errors that introduce an unstable eigenvector. The uniform grid without potential is used as a reference situation before introducing a potential and a nonuniform grid. This shows that the new stability criterion (29) really yields a tighter upper bound compared to the Courant limit, both on uniform and on nonuniform grids.

Consider a grid of $10 \mathrm{~nm} \times 10 \mathrm{~nm} \times 10 \mathrm{~nm}$ divided into $10 \times 10 \times 10$ cells. The wave function at the edge is set to zero, which corresponds to Dirichlet boundary conditions.

Consider the simplest case of a uniform grid, i.e. $\Delta x=\Delta y=\Delta z=1 \mathrm{~nm}$, with zero potential and $m^{*}=m_{\mathrm{e}}$, where $m_{\mathrm{e}}$ is the electron mass. This corresponds to an electron trapped in a 3-D infinite well. The time steps $\Delta t_{L}^{c}$ and $\Delta t_{H}^{c}$ denote the upper bounds of the time steps, calculated by (46) and (47) respectively, and $\Delta t_{L}^{\rho}$ and $\Delta t_{H}^{\rho}$, the upper bounds of the time steps calculated by (29) for the lower- and 


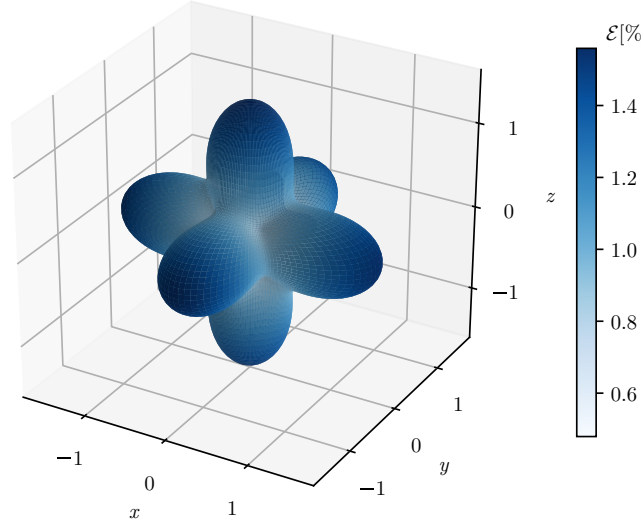

(a) Lower-order scheme.

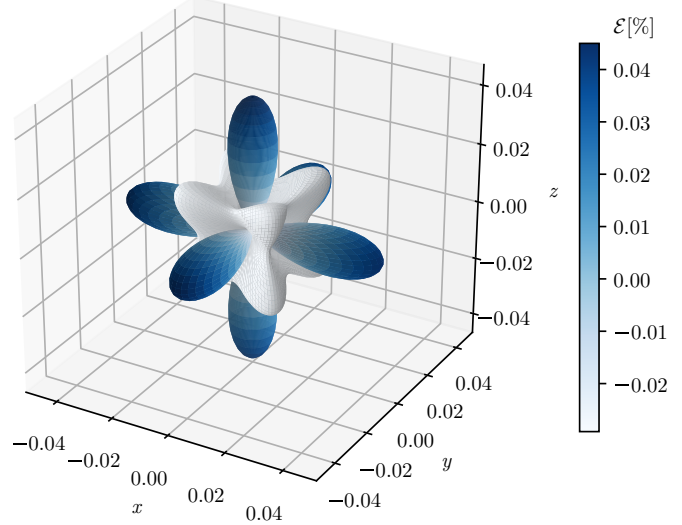

(b) Higher-order scheme.

Figure 4: Directional dependence of the dispersion error $\mathcal{E}$ (59) for both schemes when the kinetic and potential energy are equal. A discretization of 20 samples per wavelength is used and the time step is chosen at the upper bound of (51) and (52). The lower-order scheme's error is more than 30 times larger compared to the higher-order scheme.

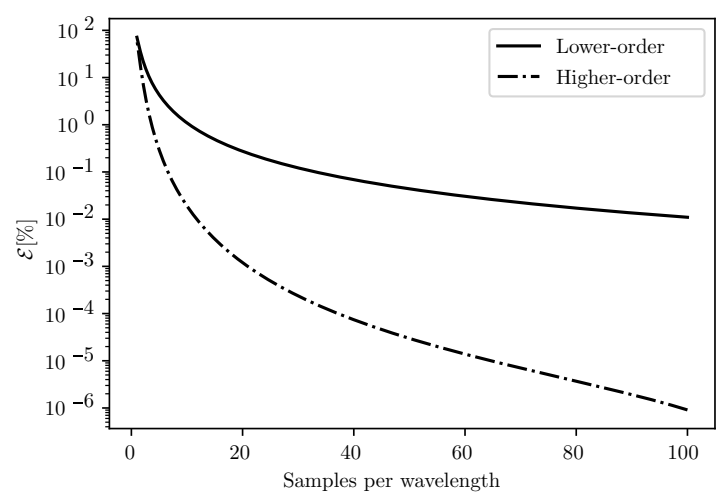

(a) Error for varying spatial steps.

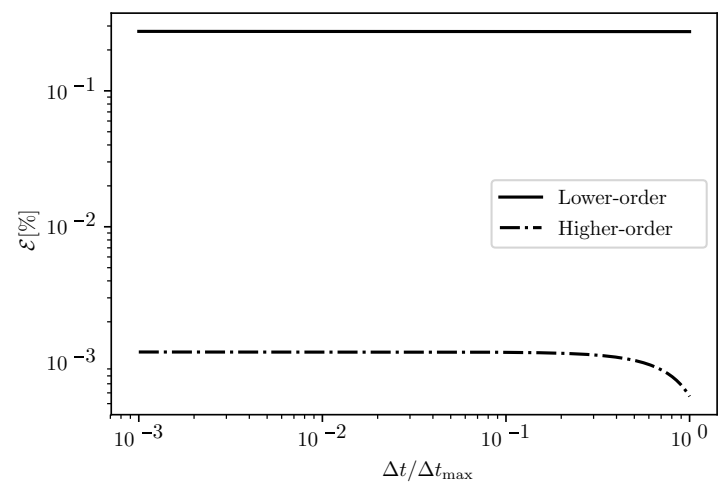

(b) Error for varying time steps.

Figure 5: The error $\mathcal{E}$ along the body diagonal, i.e. $\phi=\pi / 4$ and $\theta=\operatorname{atan}(\sqrt{2})$, as a function of (a) the spatial step at the upper bound of (51) and (52), denoted by $\Delta t_{\max }$, and (b) the time step at 20 samples per wavelength for a free particle $V=0$. 


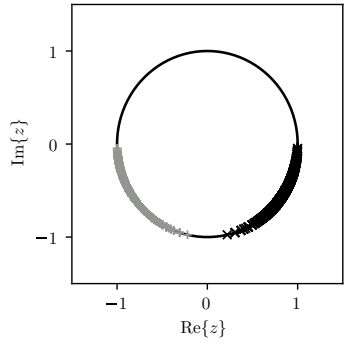

(a)

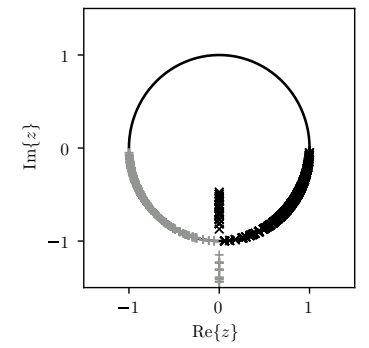

(b)

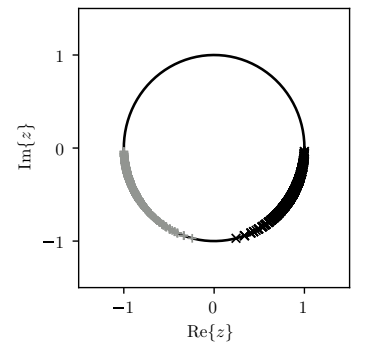

(c)

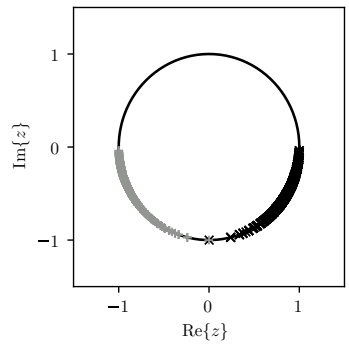

(d)

Figure 6: The eigenvalues $z$ of the iteration matrix $\mathbf{A}$ for a uniformly discretized grid and zero potential, for (a) the lowerorder scheme with time step $\Delta t=\Delta t_{L}^{c}$, (b) the higher-order scheme with the unstable time step $\Delta t=\Delta t_{L}^{c}>\Delta t_{H}^{\rho}$, (c) the higher-order scheme with time step $\Delta t=\Delta t_{H}^{c}$, and (d) the higher-order scheme with time step $\Delta t=\Delta t_{H}^{\rho}$. The black $\times$ and the grey + are the plus and minus solutions (31) and (32), respectively.

higher-order scheme, respectively. These time steps are given by:

$$
\begin{aligned}
\Delta t_{L}^{c} & =1.439665 \mathrm{fs}, & \Delta t_{L}^{\rho} & =1.475779 \mathrm{fs}, \\
\Delta t_{H}^{c} & =1.079749 \mathrm{fs}, & \Delta t_{H}^{\rho} & =1.112937 \mathrm{fs} .
\end{aligned}
$$

The eigenvalues for both proposed schemes are shown in Fig. 6 for different time steps. The eigenvalues accumulate in $z=-\jmath$ as the time step is chosen closer to the stability condition (29). Even for this very basic grid, the Courant-like stability limit is shown to be an underestimation of the true maximum time step. Because the time step in Fig. 6b is larger than $\Delta t_{H}^{\rho}$, some eigenvalues lie outside the unit circle, resulting in an unstable scheme.

The addition of a potential changes the time step. For simplicity, the case where the potential is constant, is considered. The zero point of the potential energy is arbitrary and should have no influence on any physical parameters calculated from the wave function. However, as was shown in Sections 3 and 4, both the stability criterion and the numerical dispersion error change. The time steps for a uniform potential of $V=0.3 \mathrm{eV}$ are

$$
\begin{array}{rlrl}
\Delta t_{L}^{c} & =0.8692734 \mathrm{fs}, & \Delta t_{L}^{\rho} & =0.8823096 \mathrm{fs}, \\
\Delta t_{H}^{c} & =0.7236302 \mathrm{fs}, & \Delta t_{H}^{\rho}=0.7383864 \mathrm{fs} .
\end{array}
$$

Fig. 7 reveals that the behavior of the eigenvalues is similar to the zero potential energy situation. Still it is observed that the potential increases the energy of the eigenstates, thus pushing the eigenvalues towards $z=-\jmath$, resulting in a decreased time step.

A negative potential does the inverse. It pushes the eigenvalues of the iteration matrix away from $z=-\jmath$ towards $z=\jmath$. This is shown in Fig. 8 for a uniform potential $V=-0.3 \mathrm{eV}$, resulting in the time steps

$$
\begin{aligned}
\Delta t_{L}^{c} & =2.194040 \mathrm{fs}, & \Delta t_{L}^{\rho} & =2.279034 \mathrm{fs}, \\
\Delta t_{H}^{c} & =1.946798 \mathrm{fs}, & \Delta t_{H}^{\rho} & =2.258646 \mathrm{fs} .
\end{aligned}
$$

Comparing Fig. 8a with Fig. 8b and Fig. $8 \mathrm{c}$ with Fig. $8 \mathrm{~d}$ shows that increasing the time step pushes the eigenvalues with positive imaginary part towards $z=\jmath$ and the eigenvalues with negative imaginary part towards $z=-\jmath$. The maximum time step is reached when either of these points is reached, as shown in Figs. $8 \mathrm{~b}$ and $8 \mathrm{~d}$. It is interesting to note that the time step is larger than when a zero potential is used. Since the zero energy point is arbitrary from a physical point of view, the efficiency of the proposed schemes can be increased by lowering the potential. Note that in this case, the time step calculated by (51) for the first scheme, which is used in [3, 13, 29], yields the incorrect time step $\Delta t=4.187156 \mathrm{fs}$, as (50) is violated. 


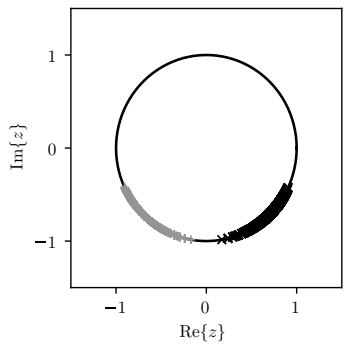

(a)

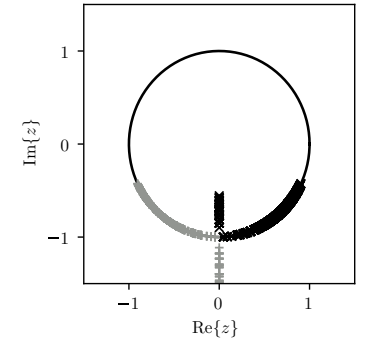

(b)

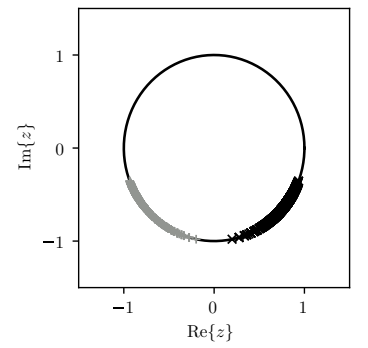

(c)

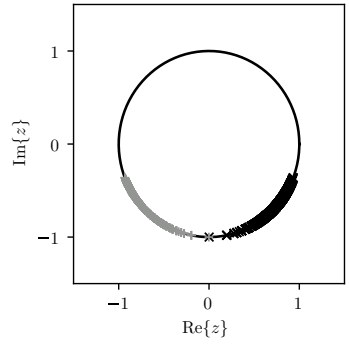

(d)

Figure 7: The eigenvalues $z$ of the iteration matrix $\mathbf{A}$ for a uniformly discretized grid and potential $V=0.3 \mathrm{eV}$, for (a) the lower-order scheme with time step $\Delta t=\Delta t_{L}^{c}$, (b) the higher-order scheme with the unstable time step $\Delta t=\Delta t_{L}^{c}>\Delta t_{H}^{\rho}$, (c) the higher-order scheme with time step $\Delta t=\Delta t_{H}^{c}$, and (d) the higher-order scheme with time step $\Delta t=\Delta t_{H}^{\rho}$. The black $\times$ and the grey + are the plus and minus solutions (31) and (32), respectively.

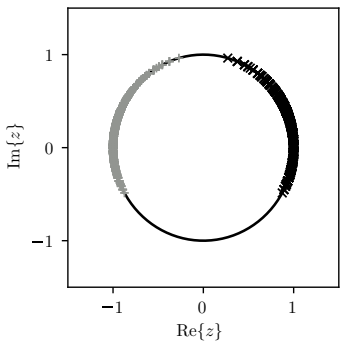

(a)

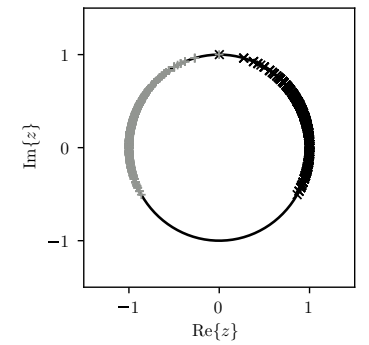

(b)

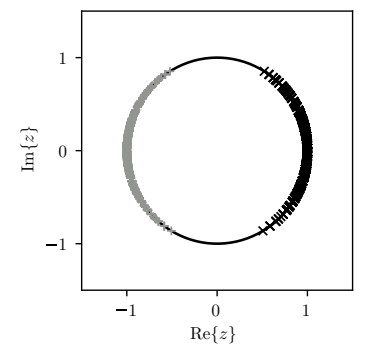

(c)

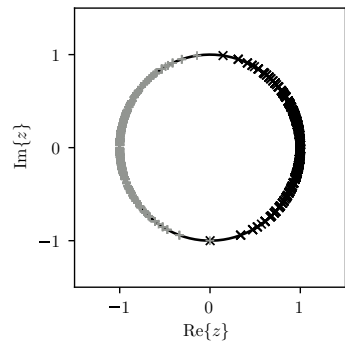

(d)

Figure 8: The eigenvalues $z$ of the iteration matrix $\mathbf{A}$ for a uniformly discretized grid and potential $V=-0.3 \mathrm{eV}$, for (a) the lower-order scheme with time step $\Delta t=\Delta t_{L}^{c}$, (b) the lower-order scheme with time step $\Delta t=\Delta t_{L}^{\rho}$, (c) the higher-order scheme with time step $\Delta t=\Delta t_{H}^{c}$, and (d) the higher-order scheme with time step $\Delta t=\Delta t_{H}^{\rho}$. The black $\times$ and the grey + are the plus and minus solutions (31) and (32), respectively.

This would lead to an unstable scheme. The resulting instability is addressed in [6], where the absolute value of $V$ is considered. However, this stability criterion leads to an underestimation of the time step, namely $\Delta t=0.8692734$ fs.

To verify whether the eigenvalues of the Hamiltonian matrix $\mathbf{H}$ are real, consider a highly nonuniform grid with randomly distributed grid points. The calculated time steps for this randomly generated grid, shown in Fig. 9a, are:

$$
\begin{array}{rlrl}
\Delta t_{L}^{c} & =1.171590 \times 10^{-2} \mathrm{fs}, & \Delta t_{L}^{\rho}=1.403755 \times 10^{-2} \mathrm{fs}, \\
\Delta t_{H}^{c}=1.601756 \times 10^{-2} \mathrm{fs}, & \Delta t_{H}^{\rho}=3.976790 \times 10^{-2} \mathrm{fs} .
\end{array}
$$

As is illustrated in Fig. 9, numerical stability is guaranteed since the eigenvalues lie on the unit circle. Moreover, for such a nonuniform grid, the Courant-like stability criterions (46) and (47) are found to be a larger underestimation of the time step. The eigenvalue plots for the lower-order scheme are omitted as they are very similar to the higher-order scheme.

\subsection{A Particle-in-a-3-D-box}

In this section, a particle-in-a-3-D-box is considered. The eigenenergies of an electron in a cavity are calculated through a long-time simulation using both schemes and compared with the analytical result. The cavity is a cube with sides $8 \mathrm{~nm}$ and the effective mass is the electron mass $m^{*}=9.1093837015 \times 10^{-31} \mathrm{~kg}=m_{\mathrm{e}}$. The cube is uniformly discretized with $\Delta x=\Delta y=\Delta z=0.2 \mathrm{~nm}$ resulting in a grid of $40 \times 40 \times 40$ cells. 


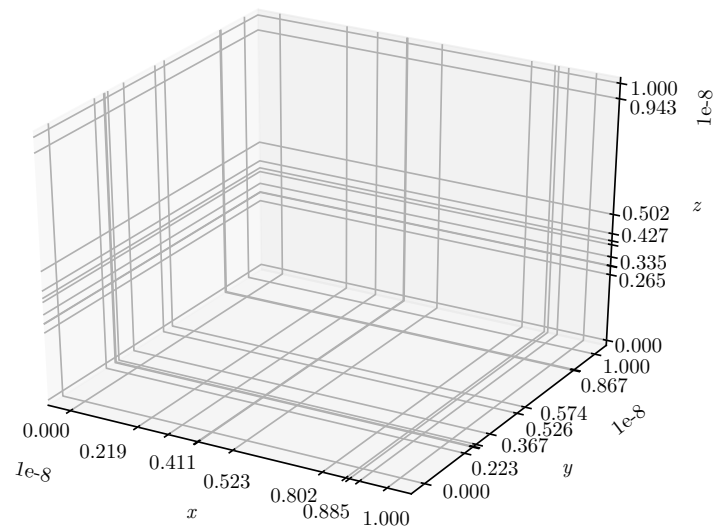

(a)

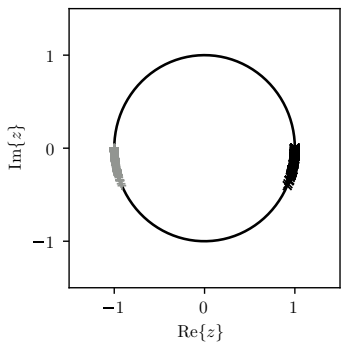

(b)

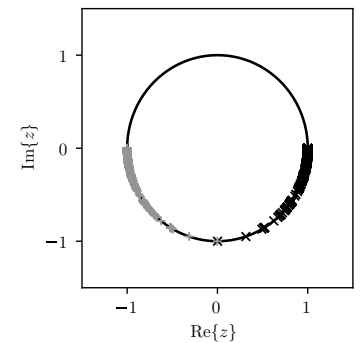

(c)

Figure 9: The eigenvalues $z$ of the iteration matrix $\mathbf{A}$ for a randomly distributed grid. (a) The randomly generated grid, (b) the eigenvalues for the higher-order scheme with time step $\Delta t=\Delta t_{H}^{c}$, and (c) the higher-order scheme with time step $\Delta t=\Delta t_{H}^{\rho}$.

The time steps calculated with the Courant-like stability criterion (39) or (40) are:

$$
\begin{aligned}
& \Delta t_{L}^{c}=57.58662 \mathrm{as}, \\
& \Delta t_{H}^{c}=43.18996 \mathrm{as} .
\end{aligned}
$$

The time steps calculated with (23) are given by

$$
\begin{aligned}
& \Delta t_{L}^{\rho}=57.67551 \text { as } \\
& \Delta t_{H}^{\rho}=43.27287 \text { as. }
\end{aligned}
$$

To determine the eigenenergies of the electron in the cavity, the method proposed in [4] is applied, where a narrow Gaussian function centered at an arbitrary position is used as the initial wavefunction. The eigenenergies are extracted from the simulation by performing a Fourier transform of the time-domain data. Instead of looking at the data in a few points, the method in [11] is applied, where the time-domain data at every point is multiplied by a random number between -0.5 and 0.5 and summed together. This is done to ensure that most eigenstates are found.

The simulation is run for $30.0 \mathrm{ps}$ using the initial wavefunction

$$
\psi(x, y, z, t=0)=\left(\frac{1}{\pi \sigma}\right)^{3 / 4} \exp \left(-\frac{\left(x-x^{\prime}\right)^{2}+\left(y-y^{\prime}\right)^{2}+\left(z-z^{\prime}\right)^{2}}{2 \sigma^{2}}\right),
$$

with $\sigma=0.5 \mathrm{~nm}, x^{\prime}=1.0 \mathrm{~nm}, y^{\prime}=-2.0 \mathrm{~nm}$ and $z^{\prime}=-0.5 \mathrm{~nm}$. The used time steps are (70) and (71), resulting in 520151 and 693274 iterations for the lower- and higher-order scheme, respectively. A Hamming window is applied to the time-domain signal to ensure that the peaks are well resolved.

In Fig. 10, the spectrum is shown for both the lower- and higher-order schemes. The values for the peaks are compared to the analytical values in Table 1. It can be seen that the higher-order scheme yields eigenenergies with a much higher accuracy. In fact, a large part of the error is due to the limited resolution of the Fourier transform. The simulation of this resonant cavity problem over many time steps confirms the accuracy of the proposed methods particularly of the higher-order scheme, which exhibits a very low numerical dispersion.

\subsection{Coherent states}

In this section, a simulation of a coherent state is performed. Modeling the behavior of a coherent state over a long time is quite challenging as many energy eigenstates propagate simultaneously with different 


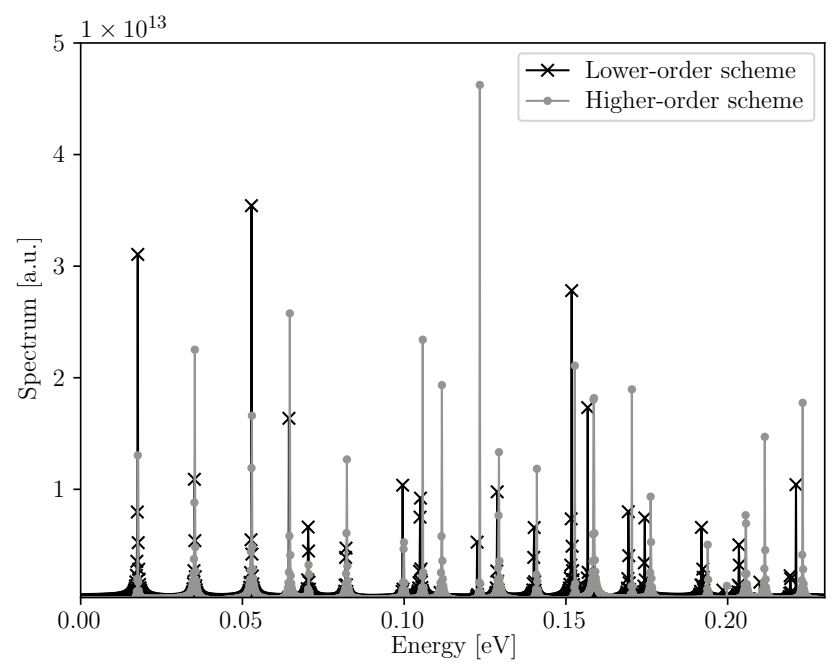

Figure 10: Comparison of the spectrum calculated with the lower-order and higher-order schemes.

\begin{tabular}{|c|c|c|c|c|c|}
\hline Mode number $n_{1} n_{2} n_{3}$ & Analytical $[\mathrm{eV}]$ & Lower-order $[\mathrm{eV}]$ & Error $[\%]$ & Higher-order $[\mathrm{eV}]$ & Error $[\%]$ \\
\hline 111 & 0.0176264 & 0.0176454 & 0.11 & 0.0176455 & 0.11 \\
\hline 211 & 0.0352528 & 0.0351529 & 0.28 & 0.0352910 & 0.11 \\
\hline 221 & 0.0528792 & 0.0527983 & 0.15 & 0.0529365 & 0.11 \\
\hline 311 & 0.0646302 & 0.0643781 & 0.39 & 0.0646543 & 0.04 \\
\hline 222 & 0.0705057 & 0.0703059 & 0.28 & 0.0704442 & 0.09 \\
\hline 321 & 0.0822566 & 0.0820235 & 0.28 & 0.0822998 & 0.05 \\
\hline 322 & 0.0998830 & 0.0995310 & 0.35 & 0.0999453 & 0.06 \\
\hline 411 & 0.1057585 & 0.1050452 & 0.67 & 0.1057353 & 0.02 \\
\hline 331 & 0.1116340 & 0.1111108 & 0.47 & 0.1116631 & 0.03 \\
\hline 421 & 0.1233849 & 0.1225528 & 0.67 & 0.1233808 & 0.00 \\
\hline 332 & 0.1292604 & 0.1287562 & 0.39 & 0.1293086 & 0.04 \\
\hline 422 & 0.1410113 & 0.1401981 & 0.58 & 0.1410263 & 0.01 \\
\hline 431 & 0.1527623 & 0.1517779 & 0.64 & 0.1527440 & 0.01 \\
\hline 511 & 0.1586377 & 0.1567407 & 1.20 & 0.1586718 & 0.02 \\
\hline 333 & 0.1586377 & 0.1578436 & 0.50 & 0.1586718 & 0.02 \\
\hline 432 & 0.1703887 & 0.1692855 & 0.65 & 0.1703896 & 0.00 \\
\hline 521 & 0.1762641 & 0.1743861 & 1.07 & 0.1761795 & 0.05 \\
\hline 522 & 0.1938906 & 0.1918936 & 1.03 & 0.1938250 & 0.03 \\
\hline 441 & 0.1938906 & 0.1923072 & 0.82 & 0.1938250 & 0.03 \\
\hline 433 & 0.1997660 & 0.1985107 & 0.63 & 0.1997528 & 0.01 \\
\hline 531 & 0.2056415 & 0.2034734 & 1.05 & 0.2055428 & 0.05 \\
\hline 442 & 0.2115170 & 0.2099526 & 0.74 & 0.2114705 & 0.02 \\
\hline 611 & 0.2232679 & 0.2193267 & 1.77 & 0.2231883 & 0.04 \\
\hline 532 & 0.2232679 & 0.2211188 & 0.96 & 0.2231883 & 0.04 \\
\hline
\end{tabular}

Table 1: Comparison of the calculated and the analytical eigenvalues. The first and second columns show the different eigenfunction modes and their eigenenergy, respectively. The third and fourth column present the eigenvalues and the error calculated with the lower-order scheme. The eigenvalues and error calculated with the higher-order scheme are shown in the last two columns. Note that for the lower-order scheme modes 511 and 333 have the same analytical eigenenergy but different calculated eigenenergies. It was assumed that the largest error corresponds to the mode with the highest maximum mode number, i.e. 511. The same is true for eigenmodes 522 and 441 and for 611 and 532 . The error of the higher-order scheme is so small that the modes remain indistinguishable. 
amplitudes. Small errors in the time evolution and amplitude of each eigenstate will gradually add up and result in decoherence. As such, the accuracy of the schemes is very visible, even for this highly dynamical simulation, since both the expectation value of the position and the shape of the probability distribution can be compared with the analytic result. The efficiency and accuracy of the higher-order scheme is compared with the lower-order scheme and the nonuniform grid is evaluated based on the speedup with respect to uniform grids.

A grid of $24 \mathrm{~nm} \times 12 \mathrm{~nm} \times 12 \mathrm{~nm}$ is considered with a harmonic oscillator potential

$$
V(x, y, z)=\frac{1}{2} m^{*} \kappa^{2}\left(x^{2}+y^{2}+z^{2}\right) .
$$

The mass and angular frequency are set to $m^{*}=0.023 m_{\mathrm{e}}$ and $\kappa=1.984 \times 10^{15} \mathrm{rad} \mathrm{s}^{-1}$, respectively, where $m_{\mathrm{e}}$ is the electron mass. This artificial atom model was obtained from [31] which was based on a quantum dot in [32]. As the theory about coherent states is needed in this section, a summary is provided in Appendix A. A normalized coherent state is initialized by shifting the ground state wave function to $x^{\prime}=-5.0 \mathrm{~nm}$. This corresponds to a coherent state with quantum numbers

$$
\left(\alpha_{x}, \alpha_{y}, \alpha_{z}\right)=(-2.2197,0,0)
$$

This results in the initial state

$$
\psi(x, y, z, t=0)=\left(\frac{m^{*} \kappa}{\pi \hbar}\right)^{3 / 4} \exp \left(-\frac{m^{*} \kappa}{2 \hbar}\left(\left(x-x^{\prime}\right)^{2}+y^{2}+z^{2}\right)\right) .
$$

The accuracy of the two proposed schemes is evaluated for this set-up by comparing the analytical expectation value of the position $\langle\hat{x}(t)\rangle_{\text {ana }}$ (A.6) to the numerically determined expectation value $\langle\hat{x}(t)\rangle_{\text {num }}$ using the error measure

$$
\mathcal{E}_{\text {coh }}=\frac{1}{\langle\hat{x}\rangle_{\max }} \sqrt{\frac{1}{T} \int_{0}^{T}\left(\langle\hat{x}(\tau)\rangle_{\text {ana }}-\langle\hat{x}(\tau)\rangle_{\text {num }}\right)^{2} \mathrm{~d} \tau},
$$

where $\langle\hat{x}\rangle_{\max }=\max _{t}\langle\hat{x}(t)\rangle_{\text {ana }}=5.0 \mathrm{~nm}$. The numerical expectation value of the position is determined by calculating the integral

$$
\langle\hat{x}(t)\rangle_{\text {num }}=\iiint \psi^{*} x \psi \mathrm{d} x \mathrm{~d} y \mathrm{~d} z .
$$

Since the wave function is normalized at initialization, it is not necessary to divide by the norm of the wave function and, as such, deviations in the conservation of the norm contribute to the overall error. The integrals in (76) and (77) are evaluated numerically by means of the trapezoidal rule.

In Table 2 the parameters of all the simulations performed in this section are provided. The first index of the simulation number indicates the used scheme, either $L$ or $H$ for the lower- and higher-order schemes, respectively, and the second index indicates the simulation number. Simulations $(L, 1)-(H, 3)$ are performed using a uniform grid such that the lower-order scheme is second-order accurate and the higher-order scheme is fourth-order accurate. The time step $\Delta t$ is calculated using the Courant-like stability criterion (51) or (52). Simulations $\left(L^{\star}, 1\right)-\left(H^{\star}, 2\right)$ are performed using a nonuniform grid, with grid points along the $x$-direction given by:

$$
x_{i}= \begin{cases}a_{1}\left(i-b_{1}\right)^{3}, & i<25 \\ a_{2} i+b_{2}, & 25 \leq i<71 \\ a_{3}\left(i-b_{3}\right)^{3}, & \text { else }\end{cases}
$$

where the parameters are defined in Table 3. Along the $y$ - and $z$-directions, the cell sizes are scaled from the center outward by a constant grading ratio of 1.05 such that each cell is scaled by a factor 1.05 compared to the adjacent cell closer to the center. The cell sizes denoted in Table 2 correspond to the smallest cell size. 


\begin{tabular}{|l|r|r|r|r|r|r|r||r|r|r|}
\hline Sim. & $\Delta x[\mathrm{~nm}]$ & $n_{x}$ & $\Delta y[\mathrm{~nm}]$ & $n_{y}$ & $\Delta z[\mathrm{~nm}]$ & $n_{z}$ & $\Delta t[\mathrm{as}]$ & CPU time $[\mathrm{s}]$ & $\mathcal{E}_{\text {coh }}[\%]$ & OOC \\
\hline \hline$(L, 1)$ & 0.3 & 80 & 0.3 & 40 & 0.3 & 40 & 2.44 & 113 & 49.4 & - \\
\hline$(L, 2)$ & 0.2 & 120 & 0.2 & 60 & 0.2 & 60 & 1.20 & 785 & 23.4 & 1.843 \\
\hline$(L, 3)$ & 0.1 & 240 & 0.1 & 120 & 0.1 & 120 & 0.32 & 24225 & 5.94 & 1.928 \\
\hline \hline$(H, 1)$ & 0.3 & 80 & 0.3 & 40 & 0.3 & 40 & 1.93 & 181 & 3.03 & - \\
\hline$(H, 2)$ & 0.2 & 120 & 0.2 & 60 & 0.2 & 60 & 0.92 & 1250 & 0.504 & 4.424 \\
\hline$(H, 3)$ & 0.1 & 240 & 0.1 & 120 & 0.1 & 120 & 0.24 & 34111 & 0.0292 & 4.225 \\
\hline \hline$\left(L^{\star}, 1\right)$ & 0.174 & 97 & 0.2 & 36 & 0.2 & 36 & 1.21 & 208 & 27.0 & - \\
\hline$\left(L^{\star}, 2\right)$ & 0.174 & 97 & 0.2 & 36 & 0.2 & 36 & 1.26 & 199 & 27.0 & - \\
\hline$\left(H^{\star}, 1\right)$ & 0.174 & 97 & 0.2 & 36 & 0.2 & 36 & 0.92 & 319 & 0.388 & - \\
\hline$\left(H^{\star}, 2\right)$ & 0.174 & 97 & 0.2 & 36 & 0.2 & 36 & 0.96 & 309 & 0.376 & - \\
\hline
\end{tabular}

Table 2: The parameters for the performed simulations. The first index indicates the used scheme, either $L$ or $H$ for the lower- and higher-order schemes, respectively, and the second index indicates the simulation number. The ${ }^{\star}$ indicates that a nonuniform grid was used. For the latter, the grid points in the $x$-direction are given by (78). In the $y$ - and $z$-directions, the cell sizes are scaled by a constant grading ratio of 1.05 such that each cell is scaled by a factor 1.05 compared to the adjacent cell toward the center. The given cell sizes correspond to the smallest cell sizes. The time steps are calculated using the approximations (46) and (47), except for simulations $\left(L^{\star}, 2\right)$ and $\left(H^{\star}, 2\right)$ where the time step is calculated based on the upper bound of (29). In the one but last column, the error according to (76) is given for a maximum deflection of $\langle\hat{x}\rangle_{\max }=5.0 \mathrm{~nm}$. In the rightmost column, the order of convergence (OOC) is given - where applicable - using (79).

\begin{tabular}{|l|l||l|l||l|l|}
\hline$a_{1}$ & $b_{1}$ & $a_{2}$ & $b_{2}$ & $a_{3}$ & $b_{3}$ \\
\hline $2.214 \times 10^{-5} \mathrm{~nm}$ & 81.53 & $0.1739 \mathrm{~nm}$ & $-8.348 \mathrm{~nm}$ & $1.969 \times 10^{-5} \mathrm{~nm}$ & 12.21 \\
\hline
\end{tabular}

Table 3: Parameters for the discretization of the nonuniform grid in the $x$-direction defined by (78).

\begin{tabular}{|l|l|l|}
\hline Simulations & Gridfile & Potential energy file \\
\hline \hline$(L, 1),(H, 1)$ & uniform_1.txt & uniform_1potential.txt \\
\hline$(L, 2),(H, 2)$ & uniform_2.txt & uniform_2potential.txt \\
\hline$(L, 3),(H, 3)$ & uniform_3.txt & uniform_3potential.txt \\
\hline$\left(L^{\star}, 1\right),\left(L^{\star}, 2\right),\left(H^{\star}, 1\right),\left(H^{\star}, 2\right)$ & nonuniform.txt & nonuniformpotential.txt \\
\hline
\end{tabular}

Table 4: Gridfiles and potential energy files used in the simulation of the coherent state from [30]. 


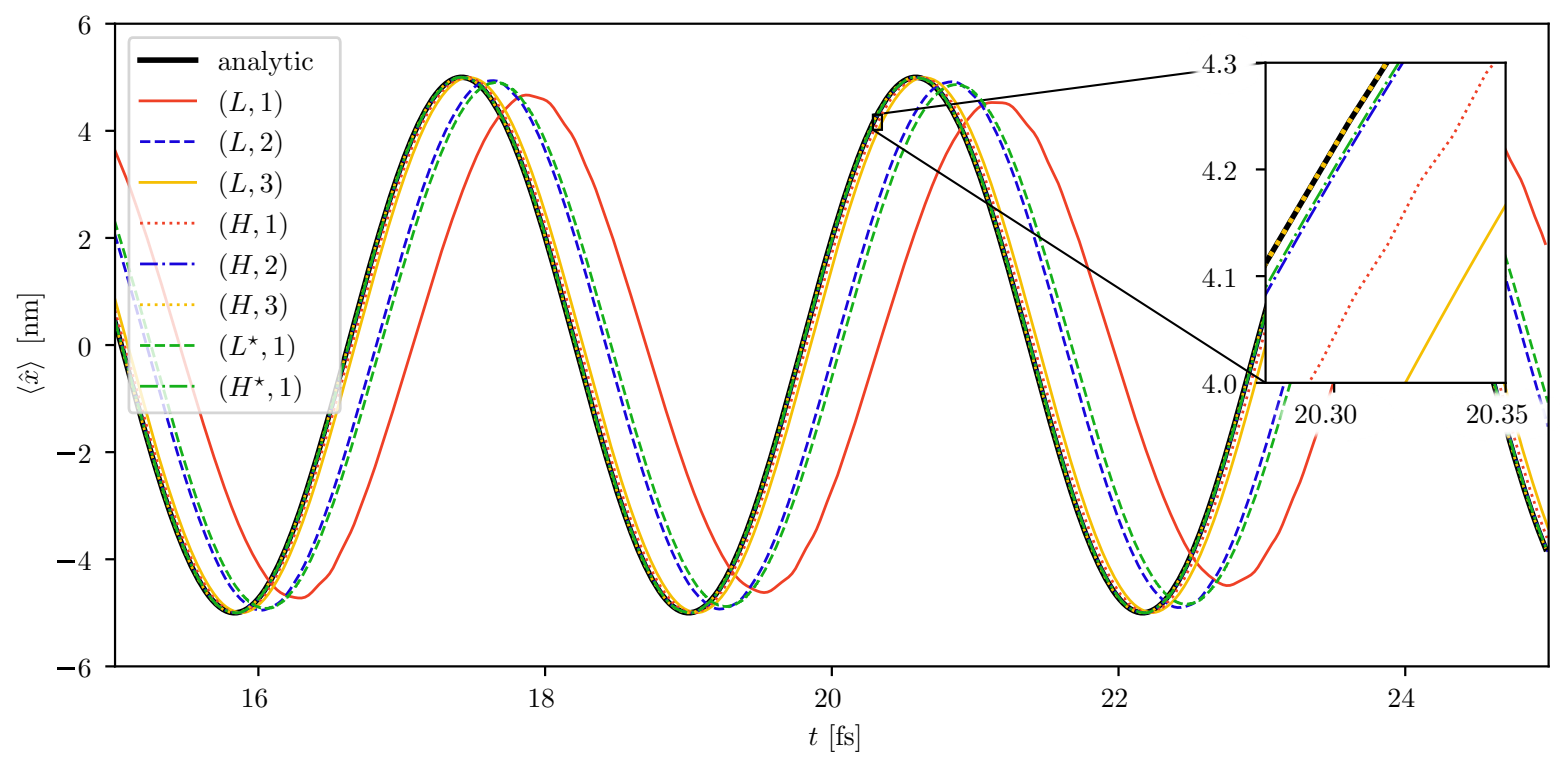

Figure 11: The analytical and the calculated expectation value of the position from $t=15 \mathrm{fs}$ to $t=25 \mathrm{fs}$. The first index of the label refers to the used scheme, either $L$ or $H$ for the lower- or higher-order scheme, respectively, and the second index refers to the simulation number, indicated in Table 2. The inset shows a zoomed in view during a small time interval $t \in[20.28 \mathrm{fs}, 20.35 \mathrm{fs}]$ to be able to differentiate the different curves. Curves $(H, 2)$ and $(H, 3)$ coincide almost completely with the analytical curve, indicating the high-accuracy of the higher-order scheme. The curves for simulations $\left(L^{\star}, 2\right)$ and $\left(H^{\star}, 2\right)$ are omitted as they overlapped with $\left(L^{\star}, 1\right)$ and $\left(H^{\star}, 1\right)$.

Simulations $\left(L^{\star}, 1\right)$ and $\left(H^{\star}, 1\right)$ use the Courant-like stability criterion $(46)$ and $(47)$, respectively, whereas simulations $\left(L^{\star}, 2\right)$ and $\left(H^{\star}, 2\right)$ use $(29)$. The calculation of the given time steps can be performed with the script in [30]. The used grids and potential energy surfaces are also provided therein. The files corresponding to each simulation are given in Table 4 . The total simulated time is 25 fs. In Fig. 11 the expectation value for the position $\langle\hat{x}(t)\rangle$ is plotted for the simulations described in Table 2, except for simulations $\left(L^{\star}, 2\right)$ and $\left(H^{\star}, 2\right)$ because these overlap with $\left(L^{\star}, 1\right)$ and $\left(H^{\star}, 1\right)$, respectively.

The error (76) corresponding to the simulations, is given in the one but last column of Table 2. The error indicates the average distance from the analytic solution during the first $25 \mathrm{fs}$, normalized by the maximal analytical distance from the center. It can be seen that the higher-order scheme using the coarsest uniform grid $(H, 1)$ already outperforms the lower-order scheme using the finest grid $(L, 3)$, even though the latter has roughly 160 times more unknowns due to the increase in grid nodes and the superlinear decrease in time step. Focusing on accuracy, the higher-order scheme performs very well for spatial steps of $0.2 \mathrm{~nm}$ and $0.1 \mathrm{~nm}$ with an error below $1 \%$ (see $(H, 2)$ and $(H, 3))$. In the rightmost column the order of convergence (OOC) is given, which is calculated as:

$$
\text { OOC }=\frac{\log \left(\mathcal{E}_{\text {coh, fine }}\right)-\log \left(\mathcal{E}_{\text {coh, coarse }}\right)}{\log \left(\Delta x_{\text {fine }}\right)-\log \left(\Delta x_{\text {coarse }}\right)}
$$

The coarse grid is either $(L, 1)$ or $(H, 1)$, depending on the used scheme, and the fine grid is given in the leftmost column of Table 2. This method of calculating the OOC is not applicable to nonuniform grids. As expected, the lower-order scheme exhibits approximately second-order convergence and the higher-order scheme exhibits approximately fourth-order convergence.

Snapshots of the particle propagating through the computational domain are given in Fig. 12 for several of the performed simulations. Figs. 12a and 12b show snapshots from simulations $(L, 1)$ and $(H, 1)$ with 

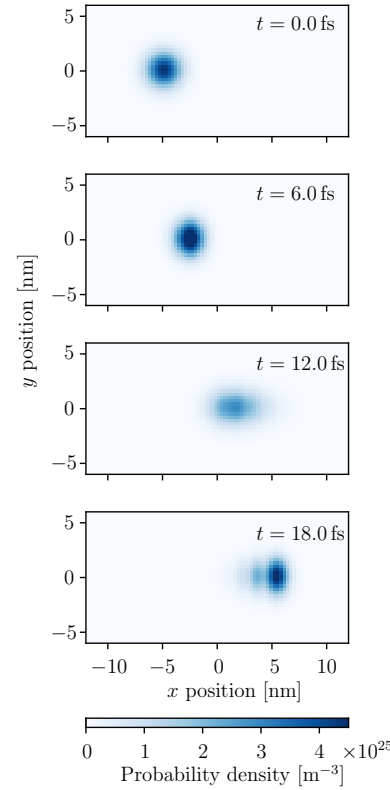

(a) $(L, 1)$
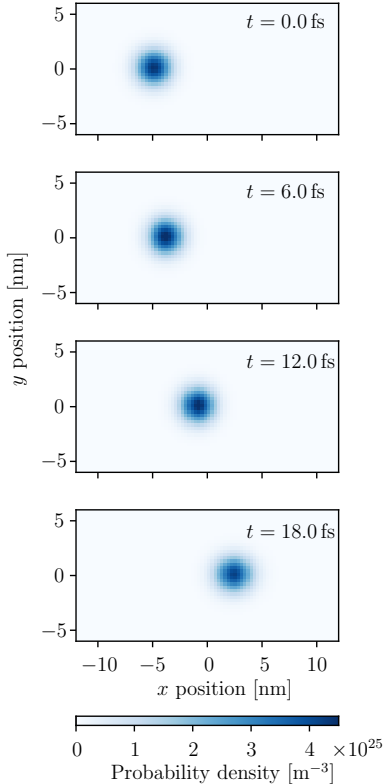

(b) $(H, 1)$
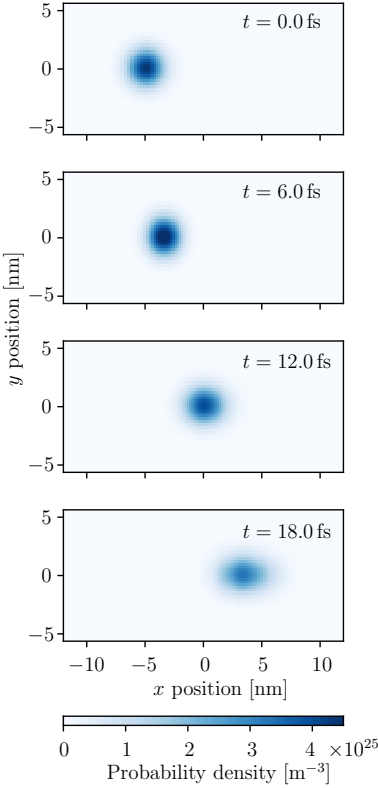

(c) $\left(L^{\star}, 1\right)$
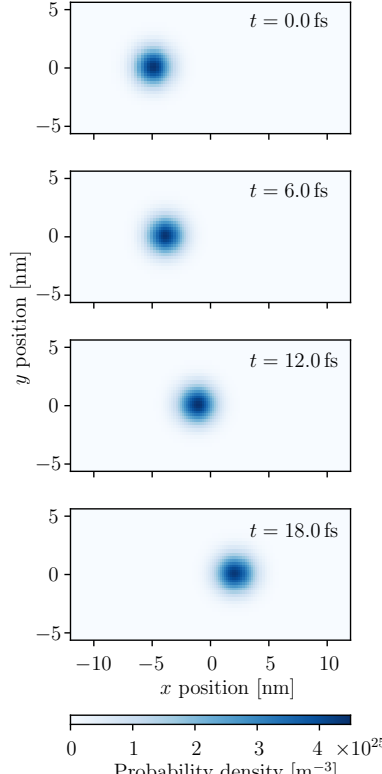
Probability density $\left[\mathrm{m}^{-3}\right]$

(d) $\left(H^{\star}, 1\right)$

Figure 12: Snapshots of the particle in a harmonic oscillator propagating through the computational domain at times $t=0.0 \mathrm{fs}$, $6.0 \mathrm{fs}, 12.0 \mathrm{fs}$ and $18.0 \mathrm{fs}$, for different simulation schemes. (a) Simulation $(L, 1)$ using the lower-order scheme on a uniform grid with $\Delta=3 \AA$, (b) simulation $(H, 1)$ using the higher-order scheme on a uniform grid with $\Delta=3 \AA$, (c) simulation $\left(L^{\star}, 1\right)$ using the lower-order scheme on the nonuniform grid, and (d) simulation $\left(H^{\star}, 1\right)$ using the higher-order scheme on the nonuniform grid.

the coarse grid, using the lower- and higher-order scheme, respectively. It is clear that the higher-order scheme is able to maintain the Gaussian probability distribution in contrast to the lower-order scheme. In Figs. 12c and 12d, the propagation of the particle through the nonuniform grid is shown for the lower- and higher-order scheme, respectively. While the lower-order scheme gradually loses its Gaussian probability distribution (Figs. 12a and 12c), the higher-order scheme perfectly maintains its shape (Figs. 12b and 12d).

The nonuniform grid decreases the computation time in two distinct ways. First, it significantly decreases the number of spatial variables, resulting in 125712 cells compared to 432000 cells for the medium uniform grid, which has approximately the same accuracy. Furthermore, it has less cells than the uniform coarse grid which contains 128000 cells. Second, the time step is increased (or at least unchanged) compared to the medium uniform grid even though the minimum cell size is decreased. These two effects are capable of reducing the CPU time by a factor 4 without compromising accuracy. In fact, the higher-order scheme using the nonuniform grid $\left(H^{\star}, 2\right)$ is even slightly more accurate than the higher-order uniform scheme $(H, 2)$ due to a decrease in cell size where necessary. As such the combination of a nonuniform grid and the rigorously derived time step allows to refine the grid intelligently. Where uniform refinement would quickly lead to an infeasible amount of calculations, a smart refinement - using a nonuniform grid and bearing (29) in mind can limit the amount of spatial variables and limit the decrease of the time step.

In Fig. 13 the propagation of the particle is shown using the nonuniform grid where the area beyond $x>0.0 \mathrm{~nm}$ is replaced with a $3 \mathrm{~nm}$ thick PML layer that is terminated with Dirichlet boundary conditions. 


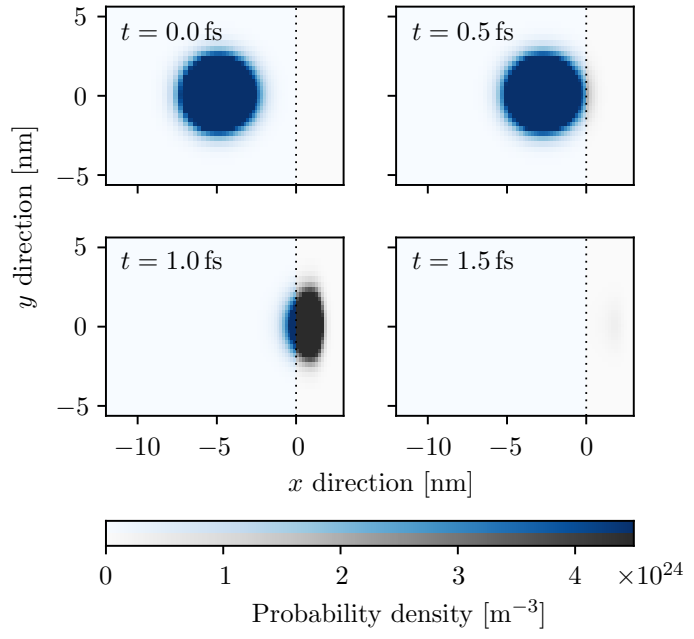

(a) Lower-order scheme

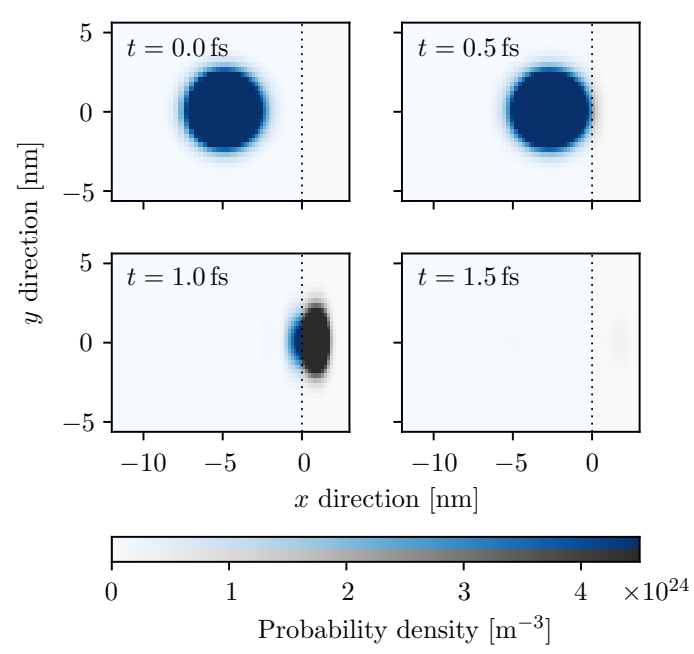

(b) Higher-order scheme

Figure 13: Snapshots of the particle inside a harmonic oscillator propagating through the computational domain at times $t=0.0 \mathrm{fs}, 0.5 \mathrm{fs}, 1.0 \mathrm{fs}$ and $1.5 \mathrm{fs}$ using a $3 \mathrm{~nm}$ thick PML layer to absorb the particle beyond $x=0.0 \mathrm{~nm}$. (a) The lower-order scheme and (b) the higher-order scheme are both able to completely absorb the particle. In order to closely observe the behavior in the PML, the color axis maximum is fixed at $4.5 \times 10^{24} \mathrm{~m}^{-3}$, which is approximately $1 / 10$ th of the maximum probability density. The cells to the right of the vertical dotted line are inside the PML and the probability density in these cells is indicated in grayscale.

The cells inside the PML are scaled as (23) and (24) with $R=\exp \left(\jmath \frac{\pi}{4}\right)$ and

$$
\sigma(x)=3.0\left(\frac{x}{d}\right)^{2}
$$

where $d=3 \mathrm{~nm}$ is the thickness of the PML layer. Update scheme (25) is used inside the PML. The snapshots are given at times $t=0.0 \mathrm{fs}, 0.5 \mathrm{fs}, 1.0 \mathrm{fs}$ and $1.5 \mathrm{fs}$, thus spanning approximately half an oscillation of the harmonic oscillator. The probability density in the cells inside the PML layer is indicated in grayscale and the boundary between the regular harmonic oscillator and the PML is depicted by the dotted line. Both the lower- and higher-order schemes are able to completely absorb the incoming particle.

\section{Conclusion}

In this paper two novel update schemes were introduced for solving the Schrödinger equation on nonuniform tensor product grids. The two schemes are first- and third-order spatially accurate, becoming second- and fourth-order accurate on uniform grids. The spatial discretization scheme can incorporate a perfectly matched layer to simulate unbounded domains. Rigorous stability conditions were derived and it was shown that these are consistent with the known results on uniform grids. It was also demonstrated that a Courant-like stability criterion yields an underestimation of the true maximum time step on uniform grids. Moreover, it is possible to shift the zero point energy of a potential in order to obtain a larger time step and increase the overall computational efficiency. The numerical dispersion error was derived for both schemes and studied as a function of the propagation direction, discretization, and the time step. The higher-order scheme was shown to exhibit stronger anisotropic behavior while increasing the accuracy substantially.

Numerical experimentation and comparison with analytical solutions have confirmed the accuracy and efficiency of the proposed methods. The higher-order accurate scheme is able to predict the dynamical behavior of a particle in a harmonic oscillator with a reasonable amount of computer resources and with 
great accuracy. Nonuniform grids were able to decrease the computation time by a factor 4 by both reducing the number of spatial unknowns and increasing the time step, and this without compromising the accuracy.

\section{Appendix A. Coherent States [33]}

A coherent state is a state of the harmonic oscillator most closely resembling the classical harmonic oscillator. The probability distribution is represented by an oscillating Gaussian distribution. It is composed of a superposition of many energy eigenstates of the harmonic oscillator, making it a broadband state. The potential for the 3-D harmonic oscillator is defined as

$$
V(x, y, z)=\frac{1}{2} m \kappa^{2}\left(x^{2}+y^{2}+z^{2}\right)
$$

where $m$ is the particle's mass and $\kappa$ the angular frequency. The eigenfunctions in 3-D are characterized by the set of three quantum numbers $\left(n_{x}, n_{y}, n_{z}\right)$ and defined in the coordinate representation by a product of the three 1-D eigenfunctions

$$
\phi_{n_{x}, n_{y}, n_{z}}(x, y, z)=\phi_{n_{x}}(x) \phi_{n_{y}}(y) \phi_{n_{z}}(z)
$$

where the 1-D eigenfunctions are given by

$$
\phi_{n_{u}}(u)=\frac{1}{\sqrt{2^{n_{u}} n_{u} !}}\left(\frac{m \kappa}{\pi \hbar}\right)^{1 / 4} e^{-\frac{m \kappa u^{2}}{2 \hbar}} H_{n_{u}}\left(\sqrt{\frac{m \kappa}{\hbar} u}\right), \quad u \in\{x, y, z\},
$$

with $H_{n_{u}}$ the Hermite polynomials. A coherent state $\chi_{\alpha_{u}}$ is defined in 1-D as the eigenvector of the creation operator with eigenvalue $\alpha_{u} \in \mathbb{C}$. The time evolution of a coherent state can be written as a superposition of the energy eigenstates

$$
\chi_{\alpha_{u}}(u, t)=e^{-\frac{1}{2}\left|\alpha_{u}\right|^{2}} e^{-\jmath \frac{\kappa t}{2}} \sum_{n_{u}} \frac{\left(\alpha_{u} e^{-\jmath \kappa t}\right)^{n_{u}}}{\sqrt{n_{u} !}} \phi_{n_{u}}(u) .
$$

It is again possible to write a coherent state in $3-\mathrm{D}$ as a product of $1-\mathrm{D}$ coherent states as

$$
\chi_{\alpha_{x}, \alpha_{y}, \alpha_{z}}(x, y, z, t)=\chi_{\alpha_{x}}(x, t) \chi_{\alpha_{y}}(y, t) \chi_{\alpha_{z}}(z, t),
$$

such that a $3-\mathrm{D}$ coherent state is characterized by the three quantum numbers $\left(\alpha_{x}, \alpha_{y}, \alpha_{z}\right)$. The ground state of the harmonic oscillator is a coherent state with $\alpha_{x}=\alpha_{y}=\alpha_{z}=0$. The expectation value of the position behaves as a classical point particle in a harmonic oscillator. For the $x$-dimension, this yields

$$
\langle\hat{x}(t)\rangle=\left|\alpha_{x}\right| \sqrt{\frac{2 \hbar}{m \kappa}} \cos \left(\varphi_{x}-\kappa t\right),
$$

where $\varphi_{x}$ is the phase of $\alpha_{x}$ as $\alpha_{x}=\left|\alpha_{x}\right| \exp \left(\jmath \varphi_{x}\right)$. Moreover, the probability density is an unchanging oscillating Gaussian distribution

$$
\left|\chi_{\alpha_{x}}\right|^{2}=\sqrt{\frac{m \kappa}{\pi \hbar}} e^{-\frac{m \kappa}{\hbar}\left(x-\sqrt{\frac{2 \hbar}{m \kappa}}\left|\alpha_{x}\right| \cos \left(\varphi_{x}-\kappa t\right)\right)^{2}} .
$$

It is possible to initialize a coherent state with real $\alpha_{x}$ by shifting the ground state wave function $\phi_{0}(x)$ to a starting position

$$
x^{\prime}=\sqrt{\frac{2 \hbar}{m \kappa}} \alpha_{x}
$$




\section{References}

[1] A. Goldberg, H. M. Schey, and J. L. Schwartz, Computer-Generated Motion Pictures of One-Dimensional QuantumMechanical Transmission and Reflection Phenomena, Am. J. Phys., vol. 35, no. 3, pp. 177-186, Mar. 1967.

[2] A. Askar and A. S. Cakmak, Explicit integration method for the time-dependent Schrodinger equation for collision problems, J. Chem. Phys., vol. 68, no. 6, pp. 2794-2798, 1978.

[3] P. B. Visscher, A fast explicit algorithm for the time-dependent Schrödinger equation, Comput. Phys., vol. 5, no. 6, p. 596, 1991.

[4] D. M. Sullivan and D. S. Citrin, Determination of the eigenfunctions of arbitrary nanostructures using time domain simulation, J. Appl. Phys., vol. 91, no. 5, pp. 3219-3226, 2002.

[5] A. Soriano, E. A. Navarro, J. A. Portí, and V. Such, Analysis of the finite difference time domain technique to solve the Schrödinger equation for quantum devices, J. Appl. Phys., vol. 95, no. 12, pp. 8011-8018, 2004.

[6] W. Dai, G. Li, R. Nassar, and S. Su, On the stability of the FDTD method for solving a time-dependent Schrödinger equation, Numer. Methods Partial Differ. Equ., vol. 21, no. 6, pp. 1140-1154, 2005.

[7] F. I. Moxley, F. Zhu, and W. Dai, A generalized FDTD method with Absorbing Boundary Condition for Solving a TimeDependent Linear Schrödinger equation, Amer. J. Comput. Math., no. 2, pp. 163-172, 2012.

[8] F. I. Moxley, T. Byrnes, F. Fujiwara, and W. Dai, A generalized finite-difference time-domain quantum method for the N-body interacting Hamiltonian, Comput. Phys. Commun., vol. 183, no. 11, pp. 2434-2440, 2012

[9] D. M. Sullivan, S. Mossman, and M. G. Kuzyk, "Time-Domain Simulation of Three Dimensional Quantum Wires," PLOS One, vol. 11, no. 4, Apr. 2016.

[10] R. Guantes and S. C. Farantos, High order finite difference algorithms for solving the Schrödinger equation in molecular dynamics, J. Chem. Phys., vol. 111, no. 24, pp. 10827-10835, 1999.

[11] N. Bigaouette, E. Ackad, and L. Ramunno, Nonlinear grid mapping applied to an FDTD-based, multi-center 3D Schrödinger equation solver, Comput. Phys. Commun., vol. 183, no. 1, pp. 38-45, 2012.

[12] Y. Xu and L. Zhang, Alternating direction implicit method for solving two-dimensional cubic nonlinear Schrödinger equation, Comput. Phys. Commun., vol. 183, no. 5, pp. 1082-1093, 2012.

[13] J. Shen, W. E. I. Sha, Z. Huang, M. Chen, and X. Wu, High-order symplectic FDTD scheme for solving a time-dependent Schrödinger equation, Comput. Phys. Commun., vol. 184, no. 3, pp. 480-492, 2013.

[14] J.-P. Berenger, A Perfectly Matched Layer for Absorption of Electromagnetic Waves, J. Comput. Phys., vol. 114, pp. 185-200, 1994.

[15] C. Zheng, A perfectly matched layer approach to the nonlinear Schrödinger wave equations, J. Comput. Phys., vol. 227, no. 1 , pp. 537-556, 2007.

[16] A. Nissen and G. Kreiss, An optimized perfectly matched layer for the Schrödinger equation, Commun. Comput. Phys., vol. 9, no. 1, pp. 147-179, 2011.

[17] D. M. Sullivan and P. M. Wilson, Time-domain determination of transmission in quantum nanostructures, J. Appl. Phys., vol. 112 , no. 6,2012 .

[18] J. F. Mennemann and A. Jüngel, Perfectly Matched Layers versus discrete transparent boundary conditions in quantum device simulations, J. Comput. Phys., vol. 275, pp. 1-24, 2014.

[19] J.-P. Berenger, Perfectly Matched Layer (PML) for Computational Electromagnetics, Morgan \& Claypool, 2007.

[20] F. I. Moxley, D. T. Chuss, and W. Dai, A generalized finite-difference time-domain scheme for solving nonlinear Schrödinger equations, Comput. Phys. Commun., vol. 184, no. 8, pp. 1834-1841, 2013.

[21] R. Eskar, P. Huang, and X. Feng, A new high-order compact ADI finite difference scheme for solving $3 D$ nonlinear Schrödinger equation, Adv. Differ. Equations, vol. 2018, no. 1, p. 286, Dec. 2018.

[22] D. S. Bernstein, Matrix Mathematics, Princeton University Press, 2009.

[23] R. F. Remis, On the Stability of the Finite-Difference Time-Domain Method, J. Comput. Phys., vol. 163, no. 1, pp. 249-261, 2000.

[24] S. Wang and F. L. Teixeira, Some Remarks on the Stability of Time-Domain Electromagnetic Simulations, IEEE Trans. Antennas Propag., vol. 52, no. 3, pp. 895-898, Mar. 2004.

[25] A. Taflove, and Susan C. Hagness, Computational Electrodynamics: The Finite-Difference Time-Domain Method (Third Edition), Artech House, 2005.

[26] B. Denecker, L. Knockaert, F. Olyslager, and D. De Zutter, A new state-space-based algorithm to assess the stability of the finite-difference time-domain method for 3D finite inhomogeneous problems, AEU - Int. J. Electron. Commun., vol. 58, no. 5, pp. 339-348, 2004.

[27] A. Van Londersele, D. De Zutter, and D. Vande Ginste, An in-depth stability analysis of nonuniform FDTD combined with novel local implicitization techniques, J. Comput. Phys., vol. 342, pp. 177-193, 2017.

[28] R. A. Horn, and C. R. Johnson Matrix analysis, Cambridge University Press, 1990.

[29] L. Pierantoni, D. Mencarelli, and T. Rozzi, A New 3-D Transmission Line Matrix Scheme for the Combined Schrödinger-Maxwell Problem in the Electronic/Electromagnetic Characterization of Nanodevices, IEEE Trans. Microw. Theory Tech., vol. 56, no. 3, pp. 654-662, Mar. 2008.

[30] P. Decleer, Nonuniform and Higher-order FDTD Methods for the Schrödinger Equation, http://sumo.intec.ugent.be/fdtd-time-step-schrodinger, 2020.

[31] C. J. Ryu, A. Y. Liu, W. E. I. Sha, and W. C. Chew, Finite-Difference Time-Domain Simulation of the Maxwell-Schrödinger System, IEEE J. Multiscale Multiphysics Comput. Tech., vol. 1, pp. 40-47, 2016.

[32] R. J. Warburton, Single spins in self-assembled quantum dots, Nat. Mater., pp. 483-493, 2013.

[33] R. A. Bertlmann, Theoretical Physics T2 Quantum Mechanics, 2008. 